Pacific

Journal of

Mathematics

\title{
FIXED POINTS OF ENDOMORPHISMS \\ OF VIRTUALLY FREE GROUPS
}

PEDRO V. SiLVA

Volume 263 No. 1

May 2013 


\title{
FIXED POINTS OF ENDOMORPHISMS OF VIRTUALLY FREE GROUPS
}

\author{
PEDRO V. SILVA
}

\begin{abstract}
A fixed point theorem is proved for inverse transducers, which leads to an automata-theoretic proof of the fixed point subgroup of an endomorphism of a finitely generated virtually free group being finitely generated. If the endomorphism is uniformly continuous for the hyperbolic metric, it is proved that the set of regular fixed points in the hyperbolic boundary has finitely many orbits under the action of the finite fixed points. In the automorphism case, it is shown that these regular fixed points are either exponentially stable attractors or exponentially stable repellers.
\end{abstract}

\section{Introduction}

Throughout the paper, the ambient groups are assumed to be finitely generated.

Gersten [1987] proved that the fixed point subgroup of a free group automorphism $\varphi$ is finitely generated. Using a different approach, Cooper [1987] gave an alternative proof, proving also that the fixed points of the continuous extension of $\varphi$ to the boundary of the free group is, in some sense, finitely generated. Bestvina and Handel [1992] achieved a major breakthrough with their innovative train track techniques, bounding the rank of the fixed point subgroup and the generating set for the infinite fixed points. Their approach was pursued by Maslakova [2003], who considered the problem of effectively computing a basis for the fixed point subgroup. The paper turned out to contain some errors, and subsequently a new paper by Bogopolski and Maslakova [2012] was posted on arXiv with the purpose of correcting these errors.

Gersten's result was generalized to further classes of groups and endomorphisms in subsequent years. Goldstein and Turner extended it to monomorphisms of free groups [1985] and to arbitrary endomorphisms [1986]. Collins and Turner extended it to automorphisms of free products of freely indecomposable groups [1994];

The author acknowledges support from the European Regional Development Fund through the COMPETE program and from the Portuguese government through Fundação para a Ciência e a Tecnologia, under the project PEst-C/MAT/UI0144/2011.

MSC2010: primary 20E05, 20E36, 20F67; secondary 68Q45, 37B25.

Keywords: virtually free groups, endomorphisms, fixed points, hyperbolic boundary, classification of fixed points. 
see the survey by Ventura [2002]. With respect to automorphisms, the widest generalization is to hyperbolic groups and is due to Paulin [1989].

Sykiotis [2002] extended Collins and Turner's result to arbitrary endomorphisms of virtually free groups using symmetric endomorphisms; see also [Sykiotis 2007] for further results on symmetric endomorphisms. In [Silva 2012], we generalized Goldstein and Turner's automata-theoretic proof to arbitrary endomorphisms of free products of cyclic groups. In the present paper, this result is extended to arbitrary endomorphisms of virtually free groups, providing an automata-theoretic alternative to Sykiotis' result.

This is done by reducing the problem to the rationality of some languages associated to a finite inverse transducer, and subsequent application of Anisimov and Seifert's theorem.

Infinite fixed points of automorphisms of free groups were discussed by Gaboriau, Jaeger, Levitt, and Lustig [Gaboriau et al. 1998], where it is remarked in particular that some of the results would hold for virtually free groups with some adaptations.

In [Silva 2010], we discussed infinite fixed points for monomorphisms of free products of cyclic groups, the group case of a more general setting based on the concept of special confluent rewriting system. These results are now extended to endomorphisms with finite kernel of virtually free groups (which are precisely the uniformly continuous endomorphisms for the hyperbolic metric), and we discuss the dynamical nature of the regular fixed points in the automorphism case, generalizing the results of [Gaboriau et al. 1998] on free groups.

The paper is organized as follows. Section 2 is devoted to preliminaries on groups and automata. We discuss inverse transducers in Section 3, proving a useful fixed point theorem. In Section 4 we prove that the fixed point subgroup is finitely generated for arbitrary endomorphisms of a (finitely generated) virtually free group $G$.

In Section 5 we get a rewriting system with good properties to represent the elements of $G$, and in Section 6 we use it to construct a simple model for the hyperbolic boundary of $G$. We study uniformly continuous endomorphisms in Section 7 and in Section 8 we prove that the infinite fixed points of such endomorphisms are, in some sense, finitely generated.

The classification of the infinite fixed points of automorphisms is performed in Section 9, and Section 10 includes an example and some open problems.

\section{Preliminaries}

Throughout the paper, we assume alphabets to be finite. We start with some grouptheoretic definitions. Given an alphabet $A$, we denote by $A^{-1}$ a set of formal inverses of $A$, and write $\widetilde{A}=A \cup A^{-1}$. We extend the mapping $a \mapsto a^{-1}$ to an 
involution of the free monoid $\widetilde{A}^{*}$ in the obvious way. As usual, the free group on $A$ is the quotient of $\widetilde{A}^{*}$ by the congruence generated by the relation $\left\{\left(a a^{-1}, 1\right): a \in \widetilde{A}\right\}$. We denote by $\theta: \widetilde{A}^{*} \rightarrow F_{A}$ the canonical morphism.

Let

$$
R_{A}=\widetilde{A}^{*} \backslash\left(\bigcup_{a \in \widetilde{A}} \widetilde{A}^{*} a a^{-1} \widetilde{A}^{*}\right)
$$

be the subset of all reduced words in $\widetilde{A}^{*}$. It is well known that, for every $g \in F_{A}$, $g \theta^{-1}$ contains a unique reduced word, denoted by $\bar{g}$. We also write $\bar{u}=\bar{u} \theta$ for every $u \in \widetilde{A}^{*}$. Note that the equivalence $u \theta=v \theta \Leftrightarrow \bar{u}=\bar{v}$ holds for all $u, v \in \widetilde{A}^{*}$.

A group $G$ is virtually free if $G$ has a free subgroup $F$ of finite index. In view of Nielsen's theorem, it is well-known that $F$ can be assumed to be normal, and is finitely generated if $G$ is finitely generated itself. Therefore every finitely generated virtually free group $G$ admits a decomposition as a disjoint union

$$
G=F \cup F b_{1} \cup \cdots \cup F b_{m},
$$

where $F \unlhd G$ is a free group of finite rank and $b_{1}, \ldots, b_{m} \in G$.

We shall need also some basic concepts from automata theory.

Let $A$ be a (finite) alphabet. A subset of $A^{*}$ is called an $A$-language. We say that $\mathscr{A}=\left(Q, q_{0}, T, \delta\right)$ is a (finite) deterministic A-automaton if

- $Q$ is a (finite) set,

- $q_{0} \in Q$ and $T \subseteq Q$,

- $\delta: Q \times A \rightarrow Q$ is a partial mapping.

We extend $\delta$ to a partial mapping $Q \times A^{*} \rightarrow Q$ by induction through

$$
(q, 1) \delta=q, \quad(q, u a) \delta=((q, u) \delta, a) \delta \quad\left(u \in A^{*}, a \in A\right) .
$$

When the automaton is clear from the context, we write $q u=(q, u) \delta$. We can view $\mathscr{A}$ as a directed graph with edges labeled by letters $a \in A$ by identifying $(p, a) \delta=q$ with the edge $p \stackrel{a}{\longrightarrow} q$. We denote by $E(\mathscr{A}) \subseteq Q \times A \times Q$ the set of all such edges.

A finite nontrivial path in $\mathscr{A}$ is a sequence

$$
p_{0} \stackrel{a_{1}}{\longrightarrow} p_{1} \stackrel{a_{2}}{\longrightarrow} \cdots \stackrel{a_{n}}{\longrightarrow} p_{n}
$$

with $\left(p_{i-1}, a_{i}, p_{i}\right) \in E(\mathscr{A})$ for $i=1, \ldots, n$. Its label is the word $a_{1} \cdots a_{n} \in A^{*}$. It is said to be a successful path if $p_{0}=q_{0}$ and $p_{n} \in T$. We also consider the trivial path $p \stackrel{1}{\longrightarrow} p$ for $p \in Q$. It is successful if $p=q_{0} \in T$.

The language $L(\mathscr{A})$ recognized by $\mathscr{A}$ is the set of all labels of successful paths in $\mathscr{A}$. Equivalently, $L(\mathscr{A})=\left\{u \in A^{*}: q_{0} u \in T\right\}$. If $\left(p_{i-1}, a_{i}, p_{i}\right) \in E(\mathscr{A})$ for every 
$i \in \mathbb{N}$, we may also consider the infinite path

$$
p_{0} \stackrel{a_{1}}{\longrightarrow} p_{1} \stackrel{a_{2}}{\longrightarrow} p_{2} \stackrel{a_{3}}{\longrightarrow} \cdots .
$$

Its label is the (right) infinite word $a_{1} a_{2} a_{3} \cdots$. We denote by $A^{\omega}$ the set of all (right) infinite words on the alphabet $A$, and also write $A^{\infty}=A^{*} \cup A^{\omega}$. We denote by $L_{\omega}(\mathscr{A})$ the set of labels of all infinite paths $q_{0} \longrightarrow \cdots$ in $\mathscr{A}$.

Given $u \in A^{*}$ and $\alpha \in A^{\infty}$, we say that $u$ is a prefix of $\alpha$ and write $u \leq \alpha$ if $\alpha=u \beta$ for some $\beta \in A^{\infty}$. By convention, this includes the case $\alpha \leq \alpha$ for $\alpha \in A^{\omega}$. For every $n \in \mathbb{N}$, we denote by $\alpha^{[n]}$ the prefix of length $n$ of $\alpha$, applying the convention that $\alpha^{[n]}=\alpha$ if $n>|\alpha|$.

It is immediate that $\left(A^{\infty}, \leq\right)$ is a complete $\wedge$-semilattice: given $\alpha, \beta \in A^{\infty}, \alpha \wedge \beta$ is the longest common prefix of $\alpha$ and $\beta$ (or $\alpha$ if $\alpha=\beta \in A^{\omega}$ ). The $\wedge$ operator will play a crucial role in later sections of the paper.

The star operator on $A$-languages is defined by

$$
L^{*}=\bigcup_{n \geq 0} L^{n}
$$

where $L^{0}=\{1\}$. An $A$-language $L$ is said to be rational if $L$ can be obtained from finite $A$-languages using finitely many times the union, product, and star operators (this is called a rational expression). Alternatively, by Kleene's theorem [Berstel 1979 , Section III], $L$ is rational if and only if it is recognized by a finite deterministic $A$-automaton $\mathscr{A}$. The definition through rational expressions generalizes to subsets of an arbitrary group in the obvious way. Moreover, if we fix a homomorphism $\pi: A^{*} \rightarrow G$, the rational subsets of $G$ are the images by $\pi$ of the rational $A$-languages. For obvious reasons, we shall be dealing mostly with matched homomorphisms. A homomorphism $\pi: \widetilde{A}^{*} \rightarrow G$ is said to be matched if $a^{-1} \pi=(a \pi)^{-1}$ for every $a \in A$. For details on rational languages and subsets, the reader is referred to [Berstel 1979; Sakarovitch 2003].

We shall need also the following classical result of Anisimov and Seifert.

Proposition 2.1 [Sakarovitch 2003, Proposition II.6.2]. Let $H$ be a subgroup of a group $G$. Then $H$ is a rational subset of $G$ if and only if $H$ is finitely generated.

We end this section with an elementary observation that helps us to establish that fixed point subgroups are finitely generated.

Proposition 2.2. Let $\pi: \widetilde{A}^{*} \rightarrow G$ be a matched epimorphism and let $X \subseteq G$. Let $A$ be a finite $\widetilde{A}$-automaton such that

(i) $L(\mathscr{A}) \subseteq X \pi^{-1}$,

(ii) $L(\mathscr{A}) \cap x \pi^{-1} \neq \varnothing$ for every $x \in X$.

Then $X$ is a rational subset of $G$. 
Proof. It follows immediately that $X=(L(\mathscr{A})) \pi$, so $X$ is a rational subset of $G$.

\section{Inverse transducers}

Given a finite alphabet $A$, we say that $\mathscr{T}=\left(Q, q_{0}, \delta, \lambda\right)$ is a (finite) deterministic A-transducer if

- $Q$ is a (finite) set,

- $q_{0} \in Q$,

- $\delta: Q \times A \rightarrow Q$ and $\lambda: Q \times A \rightarrow A^{*}$ are mappings.

As in the automaton case, we may extend $\delta$ to a mapping $Q \times A^{*} \rightarrow Q$. Similarly, we extend $\lambda$ to a mapping $Q \times A^{*} \rightarrow A^{*}$ through

$$
(q, 1) \lambda=1, \quad(q, u a) \lambda=(q, u) \lambda((q, u) \delta, a) \lambda \quad\left(u \in A^{*}, a \in A\right) .
$$

When the transducer is clear from the context, we write $q a=(q, a) \delta$. We can view $\mathcal{T}$ as a directed graph with edges labeled by elements of $A \times A^{*}$ (represented in the form $a \mid w)$ by identifying $(p, a) \delta=q,(p, a) \lambda=w$ with the edge $p \stackrel{a \mid w}{\longrightarrow} q$. The set of all such edges is denoted by $E(\mathscr{T}) \subseteq Q \times A \times A^{*} \times Q$. If $p u=q$ and $(p, u) \lambda=v$, we also write $p \stackrel{u \mid v}{\longrightarrow} q$ and call it a path in $\mathscr{T}$.

It is immediate that, given $u \in A^{*}$, there exists exactly one path in $\mathcal{T}$ of the form $q_{0} \stackrel{u \mid v}{\longrightarrow} q$. We write $u \widehat{\mathscr{T}}=v$, thus defining a mapping $\widehat{\mathscr{T}}: A^{*} \rightarrow A^{*}$.

Assume now that $\mathscr{T}=\left(Q, q_{0}, \delta, \lambda\right)$ is a deterministic $\widetilde{A}$-transducer such that $p \stackrel{a \mid u}{\longrightarrow} q$ is an edge of $\mathscr{T}$ if and only if $q \stackrel{a^{-1} \mid u^{-1}}{\longrightarrow} p$ is an edge of $\mathscr{T}$.

Then $\mathscr{T}$ is said to be inverse.

Proposition 3.1. Let $\mathscr{T}=\left(Q, q_{0}, \delta, \lambda\right)$ be an inverse $\widetilde{A}$-transducer. Then

(i) $\delta: Q \times \widetilde{A}^{*} \rightarrow Q$ induces a mapping $\hat{\delta}: Q \times F_{A} \rightarrow Q$ by $(q, u \theta) \hat{\delta}=(q, u) \delta$,

(ii) $\widehat{\mathscr{T}}: \widetilde{A}^{*} \rightarrow \widetilde{A}^{*}$ induces a mapping $\widetilde{\mathscr{T}}: F_{A} \rightarrow F_{A}$ by $u \theta \widetilde{\mathscr{T}}=u \widehat{\mathscr{T}} \theta$.

Proof. (i) Since the free group congruence $\sim$ is generated by the pairs $\left(a a^{-1}, 1\right)$, it suffices to show that $\left(q, v a a^{-1} w\right) \delta=(q, v w) \delta$ for all $q \in Q ; v, w \in \widetilde{A}^{*}$ and $a \in \widetilde{A}$.

Since $\delta$ is a full mapping, we have a path

$$
q \stackrel{v \mid v^{\prime}}{\longrightarrow} q_{1} \stackrel{a \mid u}{\longrightarrow} q_{2} \stackrel{a^{-1} \mid u^{\prime}}{\longrightarrow} q_{3} \stackrel{w \mid w^{\prime}}{\longrightarrow} q_{4}
$$

in $\mathscr{T}$. Since $\mathscr{T}$ is inverse (in particular deterministic), we must have $u^{\prime}=u^{-1}$ and $q_{3}=q_{1}$. Hence we also have a path

$$
q \stackrel{v \mid v^{\prime}}{\longrightarrow} q_{1} \stackrel{w \mid w^{\prime}}{\longrightarrow} q_{4}
$$

and so $\left(q, v a a^{-1} w\right) \delta=q_{4}=(q, v w) \delta$ as required. 
(ii) Similarly to part (i), it suffices to show that $\left(v a a^{-1} w\right) \widehat{\mathscr{T}} \theta=(v w) \widehat{\mathscr{T}} \theta$ for all $v, w \in \widetilde{A}^{*}$ and $a \in \widetilde{A}$.

We consider the path (1) for $q=q_{0}$. Since $u^{\prime}=u^{-1}$ and $q_{3}=q_{1}$, we get

$$
\left(v a a^{-1} w\right) \widehat{\mathscr{T}} \theta=\left(v^{\prime} u u^{-1} w^{\prime}\right) \theta=\left(v^{\prime} w^{\prime}\right) \theta=(v w) \widehat{\mathscr{T}} \theta,
$$

as required.

We now prove one of our main results, generalizing Goldstein and Turner's proof [1986] to mappings induced by inverse transducers.

Theorem 3.2. Let $\mathcal{T}$ be a finite inverse $\widetilde{A}$-transducer and let $z \in F_{A}$. Then

$$
L=\left\{g \in F_{A}: g \widetilde{\mathscr{T}}=g z\right\}
$$

is rational.

Proof. Write $\mathscr{T}=\left(Q, q_{0}, \delta, \lambda\right)$. For every $g \in F_{A}$, let $P_{1}(g)=g^{-1}(g \widetilde{\mathscr{T}}) \in F_{A}$ and write $q_{0} g=\left(q_{0}, g\right) \hat{\delta}, P(g)=\left(P_{1}(g), q_{0} g\right)$. Note that $g \in L$ if and only if $P_{1}(g)=z$. We define a deterministic $\widetilde{A}$-automaton $\mathscr{A}_{\mathscr{T}}=\left(P,\left(1, q_{0}\right), S, E\right)$ by

$$
\begin{aligned}
P & =\left\{P(g): g \in F_{A}\right\} ; \\
S & =P \cap(\{z\} \times Q) ; \\
E & =\left\{(P(g), a, P(g a)): g \in F_{A}, a \in \widetilde{A}\right\} .
\end{aligned}
$$

Clearly, $\mathscr{A}_{\mathcal{T}}$ is a possibly infinite automaton. Note that, since $\mathcal{T}$ is inverse, we have $q a a^{-1}=q$ for all $q \in Q$ and $a \in \widetilde{A}$. It follows that, whenever $\left(p, a, p^{\prime}\right) \in E$, $\left(p^{\prime}, a^{-1}, p\right) \in E$. We say that such edges are the inverses of each other.

Since every $w \in \widetilde{A}^{*}$ labels a unique path $P(1) \stackrel{w}{\longrightarrow} P(w \theta)$, it follows that

$$
L\left(\mathscr{A}_{\mathscr{T}}\right)=L \theta^{-1} \text {. }
$$

In view of Proposition 2.2, to prove that $L$ is rational it suffices to construct a finite subautomaton $\mathscr{B} \mathscr{T}$ of $\mathscr{A}_{\mathscr{T}}$ such that $\bar{L} \subseteq L(\mathscr{B} \mathscr{T})$.

We now fix

$$
M=\max \{|(q, a) \lambda|: q \in Q, a \in \widetilde{A}\}, \quad N=\max \{2 M+1,|z|\}
$$

and

$$
P^{\prime}=\left\{P(g) \in P:\left|P_{1}(g)\right| \leq N\right\} .
$$

Since $A$ and $\mathscr{T}$ are finite, so is $P^{\prime}$. However, infinitely many $g \in F_{A}$ may yield the same state $P(g)$.

Given $g \in F_{A}$, write $g \iota=\bar{g}^{[1]}$. Given $p=(g, q) \in P$, we also write $p \iota=g \iota$. We say that an edge $\left(p_{1}, a, p_{2}\right) \in E$ is

- central if $p_{1}, p_{2} \in P^{\prime}$,

- compatible if it is not central and $p_{1} \iota=a$. 
Lemma 3.3. (i) There are only finitely many central edges in $A_{\mathcal{T}}$.

(ii) If $\left(p_{1}, a, p_{2}\right) \in E$ is not central, either $\left(p_{1}, a, p_{2}\right)$ or $\left(p_{2}, a^{-1}, p_{1}\right)$ is compatible.

(iii) For every $p \in P$, there is at most one compatible edge leaving $p$.

Proof. (i) $A$ and $P^{\prime}$ are both finite.

(ii) Assume that $\left(p_{1}, a, p_{2}\right)$ is neither central nor compatible. Write $p_{1}=\left(g_{1}, q_{1}\right)$ and $p_{2}=\left(g_{2}, q_{2}\right)$. Suppose that $g_{1}=1$. Then $g_{2}=P_{1}(a)=a^{-1}(a \widetilde{\mathcal{T}})$ and so $\left|g_{2}\right| \leq 1+M \leq N$, in contradiction with $\left(p_{1}, a, p_{2}\right)$ being noncentral.

Thus $\bar{g}_{1}=b u$ for some $b \in \tilde{A} \backslash\{a\}$ and $u \in R_{A}$. On the other hand, we have $g_{2}=a^{-1} g_{1}\left(q_{1}, a\right) \lambda$, and so

$$
\bar{g}_{2}=\overline{a^{-1} b u\left(q_{1}, a\right) \lambda} .
$$

If $|u|<M$, then $\left|g_{1}\right|,\left|g_{2}\right| \leq 2 M+1 \leq N$ and $\left(p_{1}, a, p_{2}\right)$ is central, a contradiction. Thus $|u| \geq M \geq\left|\left(q_{1}, a\right) \lambda\right|$ and so $g_{2} \iota=a^{-1}$. Thus $\left(p_{2}, a^{-1}, p_{1}\right)$ is compatible.

(iii) Any compatible edge leaving $p$ must be labeled by $p \iota$, and $\mathscr{A}_{\mathscr{T}}$ is deterministic.

A (possibly infinite) path $q_{0} \stackrel{a_{1}}{\longrightarrow} q_{1} \stackrel{a_{2}}{\longrightarrow} \cdots$ in $\mathscr{A}_{\mathscr{T}}$ is

- central if all the vertices in it are in $P^{\prime}$,

- compatible if all the edges in it are compatible and no intermediate vertex is in $P^{\prime}$.

Lemma 3.4. Let $u \in \bar{L}$. Then there exists a path

$$
\left(1, q_{0}\right)=p_{0}^{\prime} \stackrel{u_{0}}{\longrightarrow} p_{0}^{\prime \prime} \stackrel{v_{1}}{\longrightarrow} p_{1} \stackrel{w_{1}^{-1}}{\longrightarrow} p_{1}^{\prime} \stackrel{u_{1}}{\longrightarrow} \cdots \stackrel{v_{n}}{\longrightarrow} p_{n} \stackrel{w_{n}^{-1}}{\longrightarrow} p_{n}^{\prime} \stackrel{u_{n}}{\longrightarrow} p_{n}^{\prime \prime} \in S
$$

in $A_{\mathcal{T}}$ such that

(i) $u=u_{0} v_{1} w_{1}^{-1} u_{1} \cdots v_{n} w_{n}^{-1} u_{n}$,

(ii) the paths $p_{j}^{\prime} \stackrel{u_{j}}{\longrightarrow} p_{j}^{\prime \prime}$ are central,

(iii) the paths $p_{j-1}^{\prime \prime} \stackrel{v_{j}}{\longrightarrow} p_{j}$ and $p_{j}^{\prime} \stackrel{w_{j}}{\longrightarrow} p_{j}$ are compatible,

(iv) $p_{j} \notin P^{\prime}$ if both $v_{j}$ and $w_{j}$ are nonempty.

Proof. Since $S \subseteq P^{\prime}$ by definition of $N$, there exists a path

$$
\left(1, q_{0}\right)=p_{0}^{\prime} \stackrel{u_{0}}{\longrightarrow} p_{0}^{\prime \prime} \stackrel{x_{1}}{\longrightarrow} p_{1}^{\prime} \stackrel{u_{1}}{\longrightarrow} \cdots \stackrel{x_{n}}{\longrightarrow} p_{n}^{\prime} \stackrel{u_{n}}{\longrightarrow} p_{n}^{\prime \prime} \in S
$$

in $\mathscr{A}_{\mathscr{T}}$ such that $u=u_{0} x_{1} u_{1} \cdots x_{n} u_{n}$ and the paths $p_{j}^{\prime} \stackrel{u_{j}}{\longrightarrow} p_{j}^{\prime \prime}$ (which may be trivial) collect all the occurrences of vertices in $P^{\prime}$ (and are therefore central). 
By Lemma 3.3(ii), if ( $p, a, r)$ occurs in a path $p_{j-1}^{\prime \prime} \stackrel{x_{j}}{\longrightarrow} p_{j}^{\prime}$, either $(p, a, r)$ or $\left(r, a^{-1}, p\right)$ is compatible. On the other hand, since $x_{j}$ is reduced, it follows from Lemma 3.3(iii) that $p_{j-1}^{\prime \prime} \stackrel{x_{j}}{\longrightarrow} p_{j}^{\prime}$ can be factored as

$$
p_{j-1}^{\prime \prime} \stackrel{v_{j}}{\longrightarrow} p_{j} \stackrel{w_{j}^{-1}}{\longrightarrow} p_{j}^{\prime}
$$

with $p_{j-1}^{\prime \prime} \stackrel{v_{j}}{\longrightarrow} p_{\dot{x}_{j}}$ and $p_{j}^{\prime} \stackrel{w_{j}}{\longrightarrow} p_{j}$ compatible. Clearly (iv) holds since no intermediate vertex of $p_{j-1}^{\prime \prime} \stackrel{x_{j}}{\longrightarrow} p_{j}^{\prime}$ belongs to $P^{\prime}$ by construction.

We say that a compatible path is maximal if it is infinite or cannot be extended (to the right) to produce another compatible path.

Lemma 3.5. For every $p \in P^{\prime}$, there exists in $A_{\mathscr{T}}$ a unique maximal compatible path $M_{p}$ starting at $p$.

Proof. Clearly, every compatible path can be extended to a maximal compatible path. Uniqueness follows from Lemma 3.3(iii).

We now define

$$
P_{1}^{\prime}=\left\{p \in P^{\prime}: M_{p} \text { has finitely many distinct edges }\right\}
$$

and $P_{2}^{\prime}=P^{\prime} \backslash P_{1}^{\prime}$. Hence $M_{p}$ contains no cycles if $p \in P_{2}^{\prime}$. By Lemma 3.5, if $M_{p}$ and $M_{p^{\prime}}$ intersect at vertex $r_{p p^{\prime}}$, they coincide from $r_{p p^{\prime}}$ onwards. In particular, if $M_{p}$ and $M_{p^{\prime}}$ intersect, then $p \in P_{1}^{\prime}$ if and only if $p^{\prime} \in P_{1}^{\prime}$. Let

$$
Y=\left\{\left(p, p^{\prime}\right) \in P_{2}^{\prime} \times P_{2}^{\prime}: M_{p} \text { intersects } M_{p^{\prime}}\right\} .
$$

For every $\left(p, p^{\prime}\right) \in Y$, let $M_{p} \backslash M_{p^{\prime}}$ denote the (finite) subpath $p \longrightarrow r_{p p^{\prime}}$ of $M_{p}$. In particular, if $p^{\prime}=p, M_{p} \backslash M_{p^{\prime}}$ is the trivial path at $p$.

Let $\mathscr{B}_{\mathrm{F}}$ be the subautomaton of $\mathscr{A}_{\mathscr{T}}$ containing

- all vertices in $P^{\prime}$ and all central edges,

- all vertices and edges in the paths $M_{p}\left(p \in P_{1}^{\prime}\right)$ and their inverses,

- all vertices and edges in the paths $M_{p} \backslash M_{p^{\prime}}\left(\left(p, p^{\prime}\right) \in Y\right)$ and their inverses.

It follows easily from Lemma 3.3(i) and the definitions of $P_{1}^{\prime}$ and $M_{p} \backslash M_{p^{\prime}}$ that $\mathscr{B}_{\mathcal{T}}$ is a finite subautomaton of $\mathscr{A}_{\mathcal{F}}$. As remarked before, it suffices to show that $\bar{L} \subseteq L\left(\mathscr{B}_{\mathscr{T}}\right)$.

Let $u \in \bar{L}$. Since $\mathscr{B}_{\mathscr{T}}$ contains all the central edges of $\mathscr{A}_{\mathscr{T}}$, it suffices to show that all subpaths

$$
p_{j-1}^{\prime \prime} \stackrel{v_{j}}{\longrightarrow} p_{j} \stackrel{w_{j}^{-1}}{\longrightarrow} p_{j}^{\prime}
$$

appearing in the factorization provided by Lemma 3.4 are paths in $\mathscr{B}_{\mathscr{T}}$.

Without loss of generality, we may assume that $v_{j} \neq 1$. If $w_{j}=1, p_{j-1}^{\prime \prime} \in P_{1}^{\prime}$ and we are done. Hence we may also assume that $w_{j} \neq 1$. Now, if one of the vertices $p_{j-1}^{\prime \prime}, p_{j}^{\prime}$ is in $P_{1}^{\prime}$, so is the other and we are done, since $\mathscr{B}_{\mathrm{g}}$ contains all 
the edges in the paths $M_{p}\left(p \in P_{1}^{\prime}\right)$ and their inverses. Hence we may assume that $p_{j-1}^{\prime \prime}, p_{j}^{\prime} \in P_{2}^{\prime}$. It follows that $p_{j}=r_{p_{j-1}^{\prime \prime}, p_{j}^{\prime}}$. (Since $v_{j} w_{j}^{-1} \in R_{A}$, the paths ${\underset{w}{w_{j}}}_{p_{j-1}^{\prime \prime}}$ and $M_{p_{j}^{\prime}}$ cannot meet before $p_{j}$.) Thus $p_{j-1}^{\prime \prime} \stackrel{v_{j}}{\longrightarrow} p_{j}$ is $M_{p_{j-1}^{\prime \prime}} \backslash M_{p_{j}^{\prime}}$ and $p_{j}^{\prime} \stackrel{w_{j}}{\longrightarrow} p_{j}$ is $M_{p_{j}^{\prime}} \backslash M_{p_{j-1}^{\prime \prime}}$, and so these are also paths in $\mathscr{B} \mathscr{T}$ as required.

\section{The fixed point subgroup}

We can now produce an automata-theoretic proof to Sykiotis' theorem.

Theorem 4.1 [Sykiotis 2002, Proposition 3.4]. Let $\varphi$ be an endomorphism of a finitely generated virtually free group. Then Fix $\varphi$ is finitely generated.

Proof. We consider a decomposition of $G$ as a disjoint union

$$
G=F b_{0} \cup F b_{1} \cup \cdots \cup F b_{m},
$$

where $F=F_{A} \unlhd G$ is a free group with $A$ finite and $b_{0}, \ldots, b_{m} \in G$ with $b_{0}=1$.

Let $\varphi_{0}: F_{A} \rightarrow F_{A}$ and $\eta: F_{A} \rightarrow\{0, \ldots, m\}$ be defined by

$$
g \varphi=\left(g \varphi_{0}\right) b_{g \eta} \quad\left(g \in F_{A}\right) .
$$

Since the decomposition (3) is disjoint, $g \varphi_{0}$ and $g \eta$ are both uniquely determined by $g \varphi$, and so both mappings are well defined.

Write $Q=\{0, \ldots, m\}$. For all $i \in Q$ and $a \in \widetilde{A}$, we have $b_{i}(a \varphi)=h_{i, a} b_{(i, a) \delta}$ for some (unique) $h_{i, a} \in F_{A}$ and $(i, a) \delta \in Q$. It follows that, for every $j \in Q$, $\mathscr{A}_{j}=(Q, 0, j, \delta)$ is a well-defined finite deterministic $\widetilde{A}$-automaton. We define also a finite deterministic $\widetilde{A}$-transducer $\mathscr{T}=(Q, 0, \delta, \lambda)$ by taking $(i, a) \lambda=\overline{h_{i, a}}$ for all $i \in Q$ and $a \in \widetilde{A}$.

Assume that

$$
i \stackrel{a \mid \overline{h_{i, a}}}{\longrightarrow}(i, a) \delta=j
$$

is an edge of $\mathscr{T}$. Then $b_{i}(a \varphi)=h_{i, a} b_{j}$ and so

$$
b_{i}=b_{i}(a \varphi)\left(a^{-1} \varphi\right)=h_{i, a} b_{j}\left(a^{-1} \varphi\right)=h_{i, a} h_{j, a^{-1}} b_{\left(j, a^{-1}\right) \delta} .
$$

This yields $h_{i, a} h_{j, a^{-1}}=1$ and $\left(j, a^{-1}\right) \delta=i$. Thus there is an edge

$$
j \stackrel{a^{-1}{\overline{h_{i, a}}}^{-1}}{\longrightarrow}\left(j, a^{-1}\right) \delta=i
$$

in $\mathscr{T}$ and so $\mathscr{T}$ is an inverse transducer. We claim that $\widetilde{\mathscr{T}}=\varphi_{0}$. Indeed, let $g=a_{1} \cdots a_{n}$ $\left(a_{i} \in \widetilde{A}_{i}\right)$. Then there exists a (unique) path in $\mathscr{T}$ of the form

$$
0=i_{0} \stackrel{a_{1} \mid \overline{h_{0}, a_{1}}}{\longrightarrow} i_{1} \stackrel{a_{2} \mid \overline{h_{i_{1}, a_{2}}}}{\longrightarrow} \cdots \stackrel{a_{n} \sqrt{h_{i_{n-1}, a_{n}}}}{\longrightarrow} i_{n} .
$$


Moreover, $i_{j}=\left(i_{j-1}, a_{j}\right) \delta$ for $j=1, \ldots, n$. It follows that

$$
\begin{aligned}
g \varphi & =b_{i_{0}}\left(a_{1} \varphi\right) \cdots\left(a_{n} \varphi\right)=h_{i_{0}, a_{1}} b_{i_{1}}\left(a_{2} \varphi\right) \cdots\left(a_{n} \varphi\right) \\
& =h_{i_{0}, a_{1}} h_{i_{1}, a_{2}} b_{i_{2}}\left(a_{3} \varphi\right) \cdots\left(a_{n} \varphi\right)=\cdots=h_{i_{0}, a_{1}} \cdots h_{i_{n-1}, a_{n}} b_{i_{n}}
\end{aligned}
$$

and so

$$
g \varphi_{0}=h_{i_{0}, a_{1}} \cdots h_{i_{n-1}, a_{n}}=\left(\overline{h_{i_{0}, a_{1}}} \cdots \overline{h_{i_{n-1}, a_{n}}}\right) \theta=g \widetilde{\widetilde{T}} .
$$

Thus $\widetilde{\mathscr{T}}=\varphi_{0}$.

Note that we have also shown that $g \eta=i_{n}=\left(0, a_{1} \cdots a_{n}\right) \delta$. Hence

$$
L\left(\mathscr{A}_{j}\right)=\left\{u \in \widetilde{A}^{*}: u \theta \eta=j\right\} .
$$

Next let

$$
Y=\left\{(i, j) \in Q \times Q: b_{j}\left(b_{i} \varphi\right) \in F_{A} b_{i}\right\} .
$$

For every $(i, j) \in Y$, let $z_{i, j} \in F_{A}$ be such that $b_{j}\left(b_{i} \varphi\right)=z_{i, j} b_{i}$ and define

$$
X_{i, j}=\left\{g \in F_{A}: g b_{i} \in \operatorname{Fix} \varphi \text { and } g \eta=j\right\} .
$$

We claim that $X_{i, j}$ is a rational subset of $F_{A}$ for every $(i, j) \in Y$. Indeed, $\left(g b_{i}\right) \varphi=$ $(g \varphi)\left(b_{i} \varphi\right)=\left(g \varphi_{0}\right) b_{g \eta}\left(b_{i} \varphi\right)$. Hence

$$
\begin{aligned}
X_{i, j} & =\left\{g \in F_{A}:\left(g \varphi_{0}\right) b_{j}\left(b_{i} \varphi\right)=g b_{i} \text { and } g \eta=j\right\} \\
& =\left\{g \in F_{A}:\left(g \varphi_{0}\right) z_{i, j} b_{i}=g b_{i} \text { and } g \eta=j\right\} \\
& =\left\{g \in F_{A}: g \varphi_{0}=g z_{i, j}^{-1}\right\} \cap\left\{g \in F_{A}: g \eta=j\right\} .
\end{aligned}
$$

Writing

$$
L_{i, j}=\left\{g \in F_{A}: g \varphi_{0}=g z_{i, j}^{-1}\right\},
$$

it follows from (4) that $X_{i, j}=L_{i, j} \cap\left(L\left(\mathscr{A}_{j}\right)\right) \theta$. Since $\varphi_{0}=\widetilde{\mathscr{T}}$, it follows from Theorem 3.2 that $X_{i, j}$ is an intersection of two rational subsets of $F_{A}$, and is hence rational itself; see [Berstel 1979, Corollary III.2.10].

Now it is easy to check that

$$
\text { Fix } \varphi=\bigcup_{i \in Q}\left(\bigcup\left\{X_{i, j}:(i, j) \in Y\right\}\right) b_{i} .
$$

Indeed, for every $(i, j) \in Y$, we have $X_{i, j} b_{i} \subseteq$ Fix $\varphi$ by definition of $X_{i, j}$. Conversely, let $g b_{i} \in$ Fix $\varphi$ for some $g \in F_{A}$ and $i \in Q$. Then $g b_{i}=\left(g b_{i}\right) \varphi=\left(g \varphi_{0}\right) b_{g \eta}\left(b_{i} \varphi\right)$ and so $b_{g \eta}\left(b_{i} \varphi\right) \in F_{A} b_{i}$. Hence $(i, g \eta) \in Y$. Since $g \in X_{i, g \eta}$, (5) holds. Since the $X_{i, j}$ are rational subsets of $F_{A}$ and therefore of $G$, it follows that Fix $\varphi$ is a rational subset of $G$ and is thus finitely generated by Proposition 2.1 . 
Unfortunately, our approach does not lead directly to an algorithm to compute a basis of Fix $\varphi$ (see [Bogopolski and Maslakova 2012]) because it is not clear how to decide in Section 3 whether $p \in P^{\prime}$ belongs to $P_{1}^{\prime}$ or $P_{2}^{\prime}$ and how to compute the paths $M_{p}$ and $M_{p} \backslash M_{p^{\prime}}$.

\section{A good rewriting system}

We recall that a (finite) rewriting system on $A$ is a (finite) subset $\mathscr{R}$ of $A^{*} \times A^{*}$. Given $u, v \in A^{*}$, we write $u \longrightarrow \mathscr{R}_{R} v$ if there exist $(r, s) \in \mathscr{R}$ and $x, y \in A^{*}$ such that $u=x r y$ and $v=x s y$. The reflexive and transitive closure of $\longrightarrow \curvearrowleft$ is denoted by $\longrightarrow$ *

We say that $\mathscr{R}$ is

- length-reducing if $|r|>|s|$ for every $(r, s) \in \mathscr{R}$,

- length-nonincreasing if $|r| \geq|s|$ for every $(r, s) \in \mathscr{R}$,

- noetherian if, for every $u \in A^{*}$, there is a bound on the length of a chain

$$
u \longrightarrow{ }_{\Re} v_{1} \longrightarrow{ }_{\Re} \cdots \longrightarrow_{\Re} v_{n},
$$

- confluent if, whenever $u \longrightarrow{ }_{\mathscr{R}}^{*} v$ and $u \longrightarrow_{\mathscr{R}}^{*} w$, there exists some $z \in A^{*}$ such that $v \longrightarrow{ }_{R}^{*} z$ and $w \longrightarrow{ }_{R}^{*} z$.

A word $u \in A^{*}$ is irreducible if no $v \in A^{*}$ satisfies $u \longrightarrow \mathscr{R} v$. We denote by $\operatorname{Irr} \mathscr{R}$ the set of all irreducible words in $A^{*}$ with respect to $\mathscr{R}$.

We introduce now some basic concepts and results from the theory of hyperbolic groups. For details on this class of groups, the reader is referred to [Ghys and de la Harpe 1990].

Let $\pi: \widetilde{A}^{*} \rightarrow G$ be a matched epimorphism with $A$ finite. The Cayley graph $\Gamma_{A}(G)$ of $G$ with respect to $\pi$ has vertex set $G$ and edges $(g, a, g(a \pi))$ for all $g \in G$ and $a \in \widetilde{A}$. We say that a path $p \stackrel{u}{\longrightarrow} q$ in $\Gamma_{A}(G)$ is a geodesic if it has shortest length among all the paths connecting $p$ to $q$ in $\Gamma_{A}(G)$. We denote by $\operatorname{Geo}_{A}(G)$ the set of labels of all geodesics in $\Gamma_{A}(G)$. Note that, since $\Gamma_{A}(G)$ is vertex-transitive, it is irrelevant whether or not we fix a basepoint.

The geodesic distance $d_{1}$ on $G$ is defined by taking $d_{1}(g, h)$ to be the length of a geodesic from $g$ to $h$. Given $X \subseteq G$ nonempty and $g \in G$, we define

$$
d_{1}(g, X)=\min \left\{d_{1}(g, x): x \in X\right\}
$$

A geodesic triangle in $\Gamma_{A}(G)$ is a collection of three geodesics

$$
P_{1}: g_{1} \longrightarrow g_{2}, \quad P_{2}: g_{2} \longrightarrow g_{3}, \quad P_{3}: g_{3} \longrightarrow g_{1}
$$


connecting three vertices $g_{1}, g_{2}, g_{3} \in G$. Let $V\left(P_{i}\right)$ denote the set of vertices occurring in the path $P_{i}$. We say that $\Gamma_{A}(G)$ is $\delta$-hyperbolic for some $\delta \geq 0$ if

$$
\forall g \in V\left(P_{1}\right): d_{1}\left(g, V\left(P_{2}\right) \cup V\left(P_{3}\right)\right)<\delta
$$

for every geodesic triangle $\left\{P_{1}, P_{2}, P_{3}\right\}$ in $\Gamma_{A}(G)$. If this happens for some $\delta$, we say that $G$ is hyperbolic. It is well known that the concept is independent from both alphabet and matched epimorphism, but the hyperbolicity constant $\delta$ may change. Virtually free groups are among the most important examples of hyperbolic groups.

We now use a theorem of Gilman, Hermiller, Holt, and Rees [Gilman et al. 2007] to prove the following result.

Lemma 5.1. Let $G$ be a finitely generated virtually free group. Then there exist a finite alphabet $A$, a matched epimorphism $\pi: \widetilde{A}^{*} \rightarrow G$, and a positive integer $N_{0}$ such that, for all $u \in \mathrm{Geo}_{A}(G)$ and $v \in \widetilde{A}^{*}$,

(i) there exists some $w \in \mathrm{Geo}_{A}(G)$ such that $w \pi=(u v) \pi$ and

$$
|u \wedge w| \geq|u|-N_{0}|v|
$$

(ii) there exists some $z \in \mathrm{Geo}_{A}(G)$ such that $z \pi=(v u) \pi$ and $\left|u^{-1} \wedge z^{-1}\right| \geq$ $|u|-N_{0}|v|$.

Proof. (i) By [Gilman et al. 2007, Theorem 1], there exists a finite alphabet $A$, a matched epimorphism $\pi: \widetilde{A}^{*} \rightarrow G$, and a finite length-reducing rewriting system $\mathscr{R}$ such that $\operatorname{Geo}_{A}(G)=\operatorname{Irr} \mathscr{R}$. The authors also prove that this property characterizes (finitely generated) virtually free groups.

Let $N_{0}=2 \max \{|r|:(r, s) \in \mathscr{R}\}$. Suppose that

$$
u v=w_{0} \longrightarrow w_{R} w_{1} \longrightarrow \mathscr{R} \cdots{ }_{\Re} w_{n}=w
$$

is a sequence of reductions leading to a geodesic $w$. Then $\left(w v^{-1}\right) \pi=u \pi$ and since $u$ is a geodesic we get $|u| \leq|v|+|w|$ and so $|u|-|w| \leq|v|$. On the other hand, since $\mathscr{R}$ is length-reducing, we get $|u|+|v|=|u v| \geq|w|+n$ and so $n-|v| \leq|u|-|w| \leq|v|$. Thus $n \leq 2|v|$.

Trivially, $\left|u \wedge w_{0}\right| \geq|u|$. Since $u \wedge w_{i-1} \in \operatorname{Geo}_{A}(G)$, it is immediate that $\left|u \wedge w_{i}\right|>\left|u \wedge w_{i-1}\right|-N_{0} / 2$ and so

$$
|u \wedge w|=\left|u \wedge w_{n}\right| \geq|u|-n \frac{N_{0}}{2} \geq|u|-N_{0}|v| .
$$

(ii) The inverse of a geodesic is still a geodesic. By applying (i) to $u^{-1}$ and $v^{-1}$, we get $\left(u^{-1} v^{-1}\right) \pi=x \pi$ for some $x \in \operatorname{Geo}_{A}(G)$ satisfying $\left|u^{-1} \wedge x\right| \geq\left|u^{-1}\right|-N_{0}\left|v^{-1}\right|$. Then we take $z=x^{-1}$.

We assume for the remainder of the paper that $G$ is a finitely generated virtually free group, $\pi: \widetilde{A}^{*} \rightarrow G$ a matched epimorphism, and $N_{0}$ a positive integer satisfying 
the conditions of Lemma 5.1. Since $G$ is hyperbolic, it follows from [Epstein et al. 1992, Theorem 3.4.5] that $\mathrm{Geo}_{A}(G)$ is an automatic structure for $G$ with respect to $\pi$ (see [Epstein et al. 1992] for definitions), and so the fellow traveler property holds for some constant $K_{0}>0$ (which can be taken as $2(\delta+1)$, if $\delta$ is the hyperbolicity constant). This amounts to saying that

$$
\left.\forall u, v \in \operatorname{Geo}_{A}(G): d_{1}(u \pi, v \pi) \leq 1 \Rightarrow \forall n \in \mathbb{N}: d_{1}\left(u^{[n]} \pi, v^{[n]} \pi\right) \leq K_{0}\right) .
$$

We fix a total ordering of $\widetilde{A}$. The shortlex ordering of $\widetilde{A}^{*}$ is defined by

$$
u \leq_{s l} v \text { if }\left\{\begin{array}{l}
|u|<|v|, \quad \text { or } \\
|u|=|v| \text { and } u=w a u^{\prime}, v=w b v^{\prime} \text { with } a<b \text { in } \widetilde{A} .
\end{array}\right.
$$

This is a well-known well-ordering of $\widetilde{A}^{*}$, compatible with multiplication on the left and on the right. Let

$$
L=\left\{u \in \operatorname{Geo}_{A}(G): u \leq_{s l} v \text { for every } v \in u \pi \pi^{-1}\right\} .
$$

By [Epstein et al. 1992, Theorem 2.5.1], $L$ is also an automatic structure for $G$ with respect to $\pi$, and therefore rational. We note that $L$ is factorial (a factor of a word in $L$ is still in $L$ ).

Given $g \in G$, let $\bar{g}$ denote the unique word of $L$ representing $g$. This corresponds precisely to free group reduction if $G=F_{A}$ and $\pi=\theta$. Since we shall not need free group reduction from now on, we also write $\bar{u}=\overline{u \pi}$ for every $u \in \widetilde{A}^{*}$ to simplify notation.

Theorem 5.2. Consider the finite rewriting system $\mathscr{R}^{\prime}$ on A defined by

$$
\mathscr{R}^{\prime}=\left\{(u, \bar{u}): u \in \widetilde{A}^{*},|u| \leq K_{0} N_{0}+1, u \neq \bar{u}\right\} .
$$

Then

(i) $\mathscr{R}^{\prime}$ is length-nonincreasing, noetherian and confluent,

(ii) $\operatorname{Irr} \mathscr{R}^{\prime}=L$.

Proof. (i) $\mathscr{R}^{\prime}$ is trivially length-nonincreasing, and that it is noetherian follows from

$$
(u, \bar{u}) \in \mathscr{R}^{\prime} \Rightarrow u>_{s l} \bar{u}
$$

and $\widetilde{A}^{*}$ being well-ordered by $\leq_{s l}$, plus compatibility of $\leq_{s l}$ with multiplication.

Next we show that

$$
u \longrightarrow{ }_{R^{\prime}}^{*} \bar{u} \text { holds for every } u \in \widetilde{A}^{*} .
$$

We use induction on $|u|$. The case $|u| \leq K_{0} N_{0}+1$ follows from the definition of $\mathscr{R}^{\prime}$. Hence assume that $|u|>K_{0} N_{0}+1$ and (8) holds for shorter words. Write 
$u=a v b$ with $a, b \in \widetilde{A}$. If $a v \notin L$, we have $u \longrightarrow{ }_{R^{\prime}}^{*} \overline{a v} b$ and $\bar{u}=\overline{\overline{a v}} b$. Hence $u \longrightarrow{ }_{R^{\prime}}^{*} \bar{u}$ follows from $\overline{a v} b \longrightarrow{ }_{R^{\prime}}^{*} \overline{\overline{a v} b}$. Hence we may assume that $a v \in L$.

Suppose that $u \notin \mathrm{Geo}_{A}(G)$. By Lemma 5.1(i), there exists some $w \in \operatorname{Geo}_{A}(G)$ such that $w \pi=(a v b) \pi$ and $|a v \wedge w| \geq|a v|-N_{0} \geq K_{0} N_{0}+1-N_{0}>0$. Hence we may write $w=a w^{\prime}$ and we get $(v b) \pi=\left(a^{-1} w\right) \pi=w^{\prime} \pi$. Since $\left|w^{\prime}\right|<|v b|$ due to $u \notin \operatorname{Geo}_{A}(G)$, we get $|\overline{v b}|<|v b|$, and so we may apply the induction hypothesis twice to get

$$
u=a v b \longrightarrow{ }_{R^{\prime}}^{*} a \overline{v b} \longrightarrow{ }_{R^{\prime}}^{*} \overline{a \overline{v b}}=\bar{u} .
$$

Thus we may assume that $u \in \operatorname{Geo}_{A}(G)$. We claim that $\bar{u}^{[1]}=a$. Let $p=K_{0} N_{0}+1<$ $|u|$. Since $u, \bar{u} \in \mathrm{Geo}_{A}(G)$ and $u \pi=\bar{u} \pi$, the fellow traveler property yields $d_{1}\left(u^{[p]} \pi, \bar{u}^{[p]} \pi\right) \leq K_{0}$, and so $u^{[p]} \pi=\left(\bar{u}^{[p]} x\right) \pi$ for some $x$ of length $\leq K_{0}$. Thus, by Lemma 5.1(i), there exists some $w \in \operatorname{Geo}_{A}(G)$ such that $w \pi=\left(\bar{u}^{[p]} x\right) \pi=u^{[p]} \pi$ and

$$
\left|\bar{u}^{[p]} \wedge w\right| \geq\left|\bar{u}^{[p]}\right|-N_{0}|x| \geq p-K_{0} N_{0}=1 .
$$

Hence $\bar{u}^{[1]}=w^{[1]}$. Now $a v \in L$ by assumption; hence $u^{[p]} \in L$, and so $u^{[p]}=\overline{u^{[p]}}$. Since $w \pi=u^{[p]} \pi$ and $w \in \operatorname{Geo}_{A}(G)$, we get $a=u^{[1]} \leq w^{[1]}=\bar{u}^{[1]}$ in $(\widetilde{A}, \leq)$. On the other hand, $\bar{u} \leq_{s l} u$ yields $\bar{u}^{[1]} \leq a$ in $(\widetilde{A}, \leq)$, and so $\bar{u}^{[1]}=a$ as claimed.

Now it follows easily that $\bar{u}=a \overline{a^{-1} u}=a \overline{v b}$ and the induction hypothesis yields $v b \longrightarrow{ }_{R^{\prime}}^{*} \overline{v b}$ and therefore $u=a v b \longrightarrow{ }_{R^{\prime}}^{*} a \overline{v b}=\bar{u}$. Therefore (8) holds.

Assume now that $u \longrightarrow{ }_{R^{\prime}}^{*} v$ and $u \longrightarrow{ }_{R^{\prime}}^{*} w$. By (8), we get $v \longrightarrow{ }_{R^{\prime}}^{*} \bar{v}=\bar{u}$ and $w \longrightarrow{ }_{R^{\prime}}^{*} \bar{w}=\bar{u}$. Hence $\mathscr{R}^{\prime}$ is confluent.

(ii) It follows from (8) that $\operatorname{Irr} \mathscr{R}^{\prime} \subseteq L$. The converse inclusion follows from the implication

$$
u \longrightarrow{ }_{R^{\prime}} v \Rightarrow u>_{s l} v,
$$

which follows in turn from (7).

We now establish some technical results which are useful in later sections.

Lemma 5.3. Let $u, v \in L$ and let $w \in \widetilde{A}^{*}$ be such that $v w \in \operatorname{Geo}_{A}(G)$ and $(v w) \pi=$ $u \pi$. Then $|u \wedge v| \geq|v|-K_{0} N_{0}$.

Proof. Let $k=|v|$ and write $u=u^{[k]} u^{\prime}$. Since $v=(v w)^{[k]}$, it follows from the fellow traveler property that $d_{1}\left(v \pi, u^{[k]} \pi\right) \leq K_{0}$. Hence we may write $v \pi=\left(u^{[k]} z\right) \pi$ with $|z| \leq K_{0}$. Since $u^{[k]}$ is itself a geodesic, it follows from Lemma 5.1(i) that there exists a geodesic $u^{[p]} z^{\prime}$ satisfying $\left(u^{[p]} z^{\prime}\right) \pi=\left(u^{[k]} z\right) \pi=v \pi$ and

$$
p=\left|u^{[k]} \wedge u^{[p]} z^{\prime}\right| \geq\left|u^{[k]}\right|-N_{0}|z| \geq|v|-K_{0} N_{0} .
$$

Now $v \in L$ yields $v \leq_{s l} u^{[p]} z^{\prime}$, and so $v^{[p]} \leq_{s l} u^{[p]}$. On the other hand, $u \in L$ yields $u \leq_{s l} v w$, and so $u^{[p]} \leq_{s l} v^{[p]}$. Thus $u^{[p]}=v^{[p]}$, and so $|u \wedge v| \geq p \geq|v|-K_{0} N_{0}$.

Proposition 5.4. (i) Let $u v \in L$ and let $w \in \widetilde{A}^{*}$ be such that $|v| \geq K_{0} N_{0}+N_{0}|w|$. Then $\overline{u v w}=u \overline{v w}$. 
(ii) Let $u \in \widetilde{A}^{*}$ and let $v w, v w^{\prime} \in L$. Then $\left|\overline{u v w} \wedge \overline{u v w^{\prime}}\right| \geq|v|-K_{0} N_{0}-N_{0}|u|$.

Proof. (i) Write $v=v_{1} v_{2}$ with $\left|v_{2}\right|=N_{0}|w|$. By Lemma 5.1(i), there exists some $u v_{1} z \in \mathrm{Geo}_{A}(G)$ such that $\left(u v_{1} z\right) \pi=(u v w) \pi$. Let $x=\overline{u v w}$. By Lemma 5.3, we get $\left|x \wedge u v_{1}\right| \geq\left|u v_{1}\right|-K_{0} N_{0}$. Since $\left|v_{1}\right|=|v|-\left|v_{2}\right| \geq K_{0} N_{0}, u \leq x$ and we may write $x=u y$ for some $y$. Since $L$ is factorial, we have $y \in L$. In view of $y \pi=\left(u^{-1} x\right) \pi=(v w) \pi$, we get $y=\overline{v w}$ and so $\overline{u v w}=u \overline{v w}$.

(ii) We may assume that $|v|>K_{0} N_{0}+N_{0}|u|$. Write $v=v_{1} v_{2}$ with $\left|v_{1}\right|=N_{0}|u|$. Let $x=\overline{u v_{1}}$ and write $p=|x|+\left|v_{2}\right|$. By the proof of Lemma 5.1, we have $x v_{2} w, x v_{2} w^{\prime} \in \operatorname{Geo}_{A}(G)$.

Let $y=\overline{u v w}$. Since $\left(x v_{2} w\right) \pi=y \pi$, it follows from the fellow traveler property that $d_{1}\left(\left(x v_{2}\right) \pi, y^{[p]} \pi\right) \leq K_{0}$. Hence we may write $\left(x v_{2}\right) \pi=\left(y^{[p]} s\right) \pi$ with $|s| \leq K_{0}$. Since $y^{[p]}$ is itself a geodesic, it follows from Lemma 5.1(i) that there exists a geodesic $y^{\left[p-K_{0} N_{0}\right]} s^{\prime}$ satisfying $\left(y^{\left[p-K_{0} N_{0}\right]} s^{\prime}\right) \pi=\left(y^{[p]} s\right) \pi=\left(x v_{2}\right) \pi$. To complete the proof, it suffices to show that

$$
\left|y \wedge \overline{x v_{2}}\right| \geq p-K_{0} N_{0} .
$$

Indeed, together with the corresponding inequality for $y^{\prime}=\overline{u v w^{\prime}}$, this implies

$$
\left|\overline{u v w} \wedge \overline{u v w^{\prime}}\right| \geq p-K_{0} N_{0} \geq\left|v_{2}\right|-K_{0} N_{0}=|v|-K_{0} N_{0}-N_{0}|u|
$$

and we obtain the desired inequality.

To prove (9), we consider the geodesic $y^{\left[p-K_{0} N_{0]}\right]} s^{\prime}$. Since $\left(y^{\left[p-K_{0} N_{0}\right]} s^{\prime}\right) \pi=$ $\left(x v_{2}\right) \pi$, we get $\overline{x v_{2}} \leq_{s l} y^{\left[p-K_{0} N_{0}\right]} s^{\prime}$, and so ${\overline{x v_{2}}}^{\left[p-K_{0} N_{0}\right]} \leq_{s l} y^{\left[p-K_{0} N_{0}\right]}$. On the other hand, $x v_{2} w$ is also a geodesic. Hence $y=\overline{u v w}=\overline{x v_{2} w} \leq_{s l} \overline{x v_{2}} w$ yields $y^{\left[p-K_{0} N_{0}\right]} \leq_{s l}{\overline{x v_{2}}}^{\left[p-K_{0} N_{0}\right]}$. Therefore $y^{\left[p-K_{0} N_{0}\right]}={\overline{x v_{2}}}^{\left[p-K_{0} N_{0}\right]}$, so (9) holds.

\section{A new model for the boundary}

We can now present a new model for the boundary of a finitely generated virtually free group which proves useful in studying infinite fixed points. The notion of boundary is indeed one of the important features associated to hyperbolic groups. To present it, we define a second distance in $G$ by means of the Gromov product (taking 1 as basepoint). We keep all the notation introduced in Section 5. In particular, $G$ is a finitely generated virtually free group and $L=\operatorname{Irr} \mathscr{R}^{\prime}$.

Given $g, h \in G$, we define

$$
(g \mid h)=\frac{1}{2}\left(d_{1}(1, g)+d_{1}(1, h)-d_{1}(g, h)\right) .
$$

Fix $\varepsilon>0$ such that $\varepsilon \delta \leq 1 / 5$, where $\delta$ is the hyperbolicity constant from Section 5 . Write $z=e^{\varepsilon}$ and define

$$
\rho(g, h)= \begin{cases}z^{-(g \mid h)} & \text { if } g \neq h, \\ 0 & \text { otherwise }\end{cases}
$$


for all $g, h \in G$. In general, $\rho$ is not a distance because it fails the triangular inequality. This problem is overcome by defining

$d_{2}(g, h)=\inf \left\{\rho\left(g_{0}, g_{1}\right)+\cdots+\rho\left(g_{n-1}, g_{n}\right): g_{0}=g, g_{n}=h ; g_{1}, \ldots, g_{n-1} \in G\right\}$.

By [Väisälä 2005, Proposition 5.16] (see also [Ghys and de la Harpe 1990, Proposition 7.10]), $d_{2}$ is a distance on $G$ and the inequalities

$$
\frac{1}{2} \rho(g, h) \leq d_{2}(g, h) \leq \rho(g, h)
$$

hold for all $g, h \in G$.

In general, the metric space $\left(G, d_{2}\right)$ is not complete. Its completion $\left(\widehat{G}, \hat{d}_{2}\right)$ is essentially unique, and $\partial G=\widehat{G} \backslash G$ is the boundary of $G$. The elements of the boundary admit several standard descriptions, such as equivalence classes of rays (infinite words whose finite factors are geodesics) where two rays are equivalent if the Hausdorff distance between them is finite [Ghys and de la Harpe 1990, Section 7.1]. We won't need precise definitions for these concepts or $\hat{d}_{2}$ since, as we shall see next, we can get a simpler description of $\widehat{G}$ for virtually free groups.

Lemma 6.1. There exists some $M_{0}>0$ such that, for all $g, h \in G$,

(i) $|\bar{g}| \leq|\bar{g} \wedge \overline{g h}|+K_{0} N_{0}+N_{0}|\bar{h}|$,

(ii) $d_{1}(g, h) \geq \frac{|\bar{g}|-|\bar{g} \wedge \bar{h}|}{N_{0}}-K_{0}$,

(iii) $|\bar{g} \wedge \bar{h}| \leq(g \mid h) \leq|\bar{g} \wedge \bar{h}|+M_{0}$.

Proof. (i) By applying Lemma 5.1 to the product $\bar{g} \bar{h}$, there exists some factorization $\bar{g}=v z$ and some geodesic $v w \in(g h) \pi^{-1}$ such that $|v| \geq|\bar{g}|-N_{0}|\bar{h}|$. Now we apply Lemma 5.3 to $u=\overline{g h}$ and $v w$ to get $|u \wedge v| \geq|v|-K_{0} N_{0}$. Hence

$$
|\bar{g} \wedge \overline{g h}|=|u \wedge v| \geq|v|-K_{0} N_{0} \geq|\bar{g}|-N_{0}|\bar{h}|-K_{0} N_{0} .
$$

(ii) Let $u=\bar{g} \wedge \bar{h}$. Applying (i) to $g$ and $g^{-1} h$, and in view of $d_{1}(g, h)=\left|\overline{g^{-1} h}\right|$, we get

$$
|\bar{g}| \leq|\bar{g} \wedge \bar{h}|+K_{0} N_{0}+N_{0} d_{1}(g, h) .
$$

(iii) We define $M_{0}=\delta+\left(2 \delta+1+K_{0}\right) N_{0}-1 / 2$, assuming that $\Gamma_{A}(G)$ is $\delta$-hyperbolic. Let $u=\bar{g} \wedge \bar{h}$, and write $\bar{g}=u v, \bar{h}=u w$. It is easy to check that $(g \mid h)=\frac{1}{2}\left(d_{1}(1, g)+d_{1}(1, h)-d_{1}(g, h)\right)=\frac{1}{2}\left(|u|+d_{1}(u \pi, g)+|u|+d_{1}(u \pi, h)-d_{1}(g, h)\right)$.

Since $d_{1}(g, h) \leq d_{1}(g, u \pi)+d_{1}(u \pi, h)$, we get $|\bar{g} \wedge \bar{h}|=|u| \leq(g \mid h)$.

Consider now the geodesic triangle determined by the paths

$$
P_{1}: u \pi \stackrel{v}{\longrightarrow} g, \quad P_{2}: u \pi \stackrel{w}{\longrightarrow} h, \quad P_{3}: g \stackrel{\overline{g^{-1} h}}{\longrightarrow} h .
$$


Since $\Gamma_{A}(G)$ is $\delta$-hyperbolic,

$$
d_{1}\left(q, V\left(P_{1}\right) \cup V\left(P_{2}\right)\right)<\delta \text { for every } q \in V\left(P_{3}\right) .
$$

Assume that $P_{3}: g=q_{0} \stackrel{a_{1}}{\longrightarrow} \cdots \stackrel{a_{n}}{\longrightarrow} q_{n}=h$ with $a_{i} \in \tilde{A}$. Since

$$
d_{1}\left(q_{0}, V\left(P_{1}\right)\right)=0<\delta \quad \text { and } \quad d_{1}\left(q_{n}, V\left(P_{2}\right)\right)=0<\delta,
$$

it follows from (11) that there exist some $j \in\{0, \ldots, n-1\}$ and $p_{1} \in V\left(P_{1}\right)$, $p_{2} \in V\left(P_{2}\right)$ such that $d_{1}\left(q_{j}, p_{1}\right), d_{1}\left(q_{j+1}, p_{2}\right) \leq \delta$. Since $P_{1}$ and $P_{2}$ are geodesics, we get

$$
\begin{aligned}
(g \mid h)= & \frac{1}{2}\left(d_{1}(1, g)+d_{1}(1, h)-d_{1}(g, h)\right) \\
=\frac{1}{2}(|u|+ & d_{1}\left(u \pi, p_{1}\right)+d_{1}\left(p_{1}, g\right) \\
& \left.\quad+|u|+d_{1}\left(u \pi, p_{2}\right)+d_{1}\left(p_{2}, h\right)-d_{1}\left(g, q_{j}\right)-1-d_{1}\left(q_{j+1}, h\right)\right) \\
=|\bar{g} \wedge \bar{h}|+ & \frac{1}{2}\left(d_{1}\left(u \pi, p_{1}\right)+d_{1}\left(u \pi, p_{2}\right)\right) \\
& \quad+\frac{1}{2}\left(d_{1}\left(p_{1}, g\right)-d_{1}\left(g, q_{j}\right)\right)+\frac{1}{2}\left(d_{1}\left(p_{2}, h\right)-d_{1}\left(q_{j+1}, h\right)\right)-\frac{1}{2} .
\end{aligned}
$$

Since $d_{1}\left(p_{1}, g\right) \leq d_{1}\left(p_{1}, q_{j}\right)+d_{1}\left(q_{j}, g\right) \leq \delta+d_{1}\left(q_{j}, g\right)$, we have

$$
\frac{1}{2}\left(d_{1}\left(p_{1}, g\right)-d_{1}\left(g, q_{j}\right)\right) \leq \frac{\delta}{2} .
$$

Similarly,

$$
\frac{1}{2}\left(d_{1}\left(p_{2}, h\right)-d_{1}\left(q_{j+1}, h\right)\right) \leq \frac{\delta}{2} .
$$

Out of symmetry, it suffices to show that $d_{1}\left(u \pi, p_{1}\right) \leq\left(2 \delta+1+K_{0}\right) N_{0}$.

Applying (ii) to $p_{1}$ and $p_{2}$, we get

$$
d_{1}\left(p_{1}, p_{2}\right) \geq \frac{\left|\overline{p_{1}}\right|-\left|\overline{p_{1}} \wedge \overline{p_{2}}\right|}{N_{0}}-K_{0} .
$$

Since $\overline{p_{1}}$ (respectively $\overline{p_{2}}$ ) is a prefix of $\bar{g}$ (respectively $\bar{h}$ ), it follows easily that $\overline{p_{1}} \wedge \overline{p_{2}}=u$ and $\left|\overline{p_{1}}\right|-\left|\overline{p_{1}} \wedge \overline{p_{2}}\right|=d_{1}\left(u \pi, p_{1}\right)$. Hence

$$
\begin{aligned}
& d_{1}\left(u \pi, p_{1}\right) \\
& \quad \leq\left(d_{1}\left(p_{1}, p_{2}\right)+K_{0}\right) N_{0} \leq\left(d_{1}\left(p_{1}, q_{j}\right)+d_{1}\left(q_{j}, q_{j+1}\right)+d_{1}\left(q_{j+1}, p_{2}\right)+K_{0}\right) N_{0} \\
& \quad \leq\left(2 \delta+1+K_{0}\right) N_{0} .
\end{aligned}
$$

The language $L$ introduced in (6) was noted to be rational. We recall that an automaton is said to be trim if every vertex occurs in some successful path. Let $\mathscr{A}=\left(Q, q_{0}, T, E\right)$ be a finite trim deterministic $\widetilde{A}$-automaton recognizing $L$ (for example, the minimal automaton of $L$; see [Berstel 1979]). Since $L$ is factorial, we must have $T=Q$. Let

$$
\partial L=\left\{\alpha \in \widetilde{A}^{\omega}: \alpha^{[n]} \in L \text { for every } n \in \mathbb{N}\right\} .
$$


Equivalently, since $\mathscr{A}$ is trim and deterministic and $T=Q$, we have $\partial L=L_{\omega}(\mathscr{A})$. Write $\hat{L}=L \cup \partial L$. We define a mapping $d_{3}: \hat{L} \times \hat{L} \rightarrow \mathbb{R}_{0}^{+}$by

$$
d_{3}(\alpha, \beta)= \begin{cases}2^{-|\alpha \wedge \beta|} & \text { if } \alpha \neq \beta, \\ 0 & \text { otherwise. }\end{cases}
$$

It is immediate that $d_{3}$ is a distance in $\hat{L}$. Indeed, an ultrametric distance since

$$
|\alpha \wedge \gamma| \geq \min \{|\alpha \wedge \beta|,|\beta \wedge \gamma|\}
$$

holds for all $\alpha, \beta, \gamma \in \hat{L}$. We commit a slight abuse of notation by also denoting by $d_{3}$ the restriction of $d_{3}$ to $L \times L$.

Proposition 6.2. (i) The mutually inverse mappings $\left(G, d_{2}\right) \rightarrow\left(L, d_{3}\right): g \mapsto \bar{g}$ and $\left(L, d_{3}\right) \rightarrow\left(G, d_{2}\right): u \mapsto u \pi$ are uniformly continuous;

(ii) $\left(\hat{L}, d_{3}\right)$ is the completion of $\left(L, d_{3}\right)$;

(iii) $\left(\partial L, d_{3}\right)$ is homeomorphic to the boundary of $G$.

Proof. (i) In view of (10), it suffices to show that

$$
\begin{aligned}
& \forall M>0 \exists N>0:((g \mid h)>N \Rightarrow|\bar{g} \wedge \bar{h}|>M), \\
& \forall M>0 \exists N>0:(|\bar{g} \wedge \bar{h}|>N \Rightarrow(g \mid h)>M) .
\end{aligned}
$$

Now we apply Lemma 6.1(iii).

(ii) Let $\left(\alpha_{n}\right)_{n}$ be a Cauchy sequence in $\left(\hat{L}, d_{3}\right)$. For every $k \in \mathbb{N}$, the sequence $\left(\alpha_{n}^{[k]}\right)_{n}$ stabilizes when $n \rightarrow+\infty$. Moreover, $\lim _{n \rightarrow+\infty} \alpha_{n}^{[k]}$ is a prefix of

$$
\lim _{n \rightarrow+\infty} \alpha_{n}^{[k+1]} \text {. }
$$

Let $\beta \in A^{\infty}$ be the unique word satisfying $\beta^{[k]}=\lim _{n \rightarrow+\infty} \alpha_{n}^{[k]}$ for every $k \in \mathbb{N}$. It is immediate that $\beta \in \hat{L}$ and $\beta=\lim _{n \rightarrow+\infty} \alpha_{n}$. Hence $\left(\hat{L}, d_{3}\right)$ is complete. Since $\alpha=\lim _{n \rightarrow+\infty} \alpha^{[n]}$ for every $\alpha \in \partial L,\left(\hat{L}, d_{3}\right)$ is the completion of $\left(L, d_{3}\right)$.

(iii) By (i) and (ii), the uniformly continuous mappings $\left(G, d_{2}\right) \rightarrow\left(L, d_{3}\right): g \mapsto \bar{g}$ and $\left(L, d_{3}\right) \rightarrow\left(G, d_{2}\right): u \mapsto u \pi$ admit (unique) continuous extensions to their completions (see [Dugundji 1966, Section XIV.6]), say

$$
\Phi: \widehat{G} \rightarrow \hat{L}, \quad \Psi: \hat{L} \rightarrow \widehat{G}
$$

Hence $\Phi \Psi$ is a continuous extension of the identity on $G$ to its completion $\widehat{G}$. Since such an extension is unique, $\Phi \Psi$ must be the identity mapping on $\widehat{G}$. Similarly, $\Psi \Phi$ must be the identity mapping on $\hat{L}$, and so $\Phi$ and $\Psi$ are mutually inverse homeomorphisms. Therefore the restriction $\left.\Phi\right|_{\partial G}: \partial G \rightarrow \partial L$ must also be a homeomorphism. 
We have just proved that our construction of $\hat{L}$ constitutes a model for the hyperbolic completion of $G$. But we must also import to $\hat{L}$ the algebraic operations of $\widehat{G}$ since we shall be considering homomorphisms soon. Clearly, the binary operation on $L$ is defined as

$$
L \times L \rightarrow L:(u, v) \mapsto \overline{u v},
$$

so that $\left(G, d_{2}\right) \rightarrow\left(L, d_{3}\right): g \mapsto \bar{g}$ is also a group isomorphism. But there is another important algebraic operation involved. Indeed, for every $g \in G$, the left translation $\tau_{g}: G \rightarrow G: x \mapsto g x$ is uniformly continuous for $d_{2}$ and so admits a continuous extension $\hat{\tau}_{g}: \widehat{G} \rightarrow \widehat{G}$. It follows that the left action of $G$ in its boundary, $G \times \partial G \rightarrow \partial G:(g, \alpha) \mapsto \alpha \hat{\tau}_{g}$, is continuous. We can also replicate this operation in $\hat{L}$ as follows.

Proposition 6.3. Let $u \in L$. Then $\tau_{u}: L \rightarrow L: v \mapsto \overline{u v}$ is uniformly continuous.

Proof. It suffices to show that

$$
\forall M>0 \exists N>0:(|v \wedge w|>N \Rightarrow|\overline{u v} \wedge \overline{u v}|>M) .
$$

By Proposition 5.4(ii), we can take $N=M+K_{0} N_{0}+N_{0}|u|$.

Therefore $\tau_{u}$ admits a continuous extension $\hat{\tau}_{u}: \hat{L} \rightarrow \hat{L}$ and the left action $L \times \partial L \rightarrow \partial L:(u, \alpha) \mapsto \alpha \hat{\tau}_{u}$ is continuous. Write $\overline{u \alpha}=\alpha \hat{\tau}_{u}$. For every $\alpha \in \partial L$, we have

$$
\overline{u \alpha}=\overline{u \lim _{n \rightarrow+\infty} \alpha^{[n]}}=\lim _{n \rightarrow+\infty} \overline{u \alpha^{[n]}} .
$$

Hence $\left(\hat{L}, d_{3}\right)$ serves as a model for $\left(\widehat{G}, \hat{d}_{2}\right)$ both topologically and algebraically. From now on, we pursue our work within $\left(\hat{L}, d_{3}\right)$.

\section{Uniformly continuous endomorphisms}

We keep all the notation introduced in Section 5. In particular, $G$ is a finitely generated virtually free group and $L=\operatorname{Irr} \mathscr{R}^{\prime}$. Following the program announced above, we work within $\left(\hat{L}, d_{3}\right)$.

Given an endomorphism $\varphi$ of $G$, we denote by $\bar{\varphi}$ the corresponding endomorphism of $L$ for the binary operation induced by the product in $G$, that is, $u \bar{\varphi}=\overline{(u \pi) \varphi}$. To simplify notation, we often write $u \varphi$ instead of $u \pi \varphi$ for $u \in \widetilde{A}^{*}$.

We say that $\varphi$ satisfies the bounded cancellation property if

$$
\{|u \bar{\varphi}|-|u \bar{\varphi} \wedge(u v) \bar{\varphi}|: u v \in L\}
$$

is bounded. In that case, we denote its maximum by $B_{\varphi}$. This property was considered originally for free group automorphisms by Cooper [1987].

We also fix the notation $D_{\varphi}=\max \{|\overline{a \varphi}|: a \in \widetilde{A}\}$ and recall that a homomorphism with finite kernel is called virtually injective. 
Theorem 7.1. Let $\varphi$ be a virtually injective endomorphism $\varphi$ of $G$. Then $\varphi$ satisfies the bounded cancellation property.

Proof. Suppose that $\varphi$ does not satisfy the bounded cancellation property. Then

$$
\forall m \in \mathbb{N} \exists u_{m} v_{m} \in L:\left|u_{m} \bar{\varphi}\right|-\left|u_{m} \bar{\varphi} \wedge\left(u_{m} v_{m}\right) \bar{\varphi}\right|>m .
$$

Let $X_{0}=\left(K_{0}+D_{\varphi}\right) N_{0}$. We claim that

$$
\begin{aligned}
& \forall m \in \mathbb{N} \exists u_{m}^{\prime} v_{m}^{\prime} \in L:\left(\left|u_{m}^{\prime} \bar{\varphi}\right|-\left|\left(u_{m}^{\prime} v_{m}^{\prime}\right) \bar{\varphi}\right|>m\right. \\
& \left.\quad \text { and }\left|\left(u_{m}^{\prime} v_{m}^{\prime}\right) \bar{\varphi}\right|-\left|u_{m}^{\prime} \bar{\varphi} \wedge\left(u_{m}^{\prime} v_{m}^{\prime}\right) \bar{\varphi}\right| \leq X_{0}\right) .
\end{aligned}
$$

Indeed, let $m \in \mathbb{N}$. Take $n=m+X_{0}$ and write $v_{n}=a_{1} \cdots a_{k}\left(a_{i} \in \widetilde{A}\right)$. For $i=0, \ldots, k$, let $w_{i}=\left(u_{n} a_{1} \cdots a_{i}\right) \bar{\varphi}$. Let $j$ denote the smallest $i$ such that

$$
\left|u_{n} \bar{\varphi} \wedge w_{i}\right| \leq\left|u_{n} \bar{\varphi} \wedge\left(u_{n} v_{n}\right) \bar{\varphi}\right| \text {. }
$$

Take $u_{m}^{\prime}=u_{n}$ and $v_{m}^{\prime}=a_{1} \cdots a_{j-1}$ (since $j>0$ ). Since $L$ is factorial, we have $u_{m}^{\prime} v_{m}^{\prime} \in L$.

Now, by the minimality of $j$, we get

$$
\left|u_{n} \bar{\varphi} \wedge w_{j-1}\right|>\left|u_{n} \bar{\varphi} \wedge\left(u_{n} v_{n}\right) \bar{\varphi}\right| \text {. }
$$

Since $\left|u_{n} \bar{\varphi} \wedge w_{j}\right| \leq\left|u_{n} \bar{\varphi} \wedge\left(u_{n} v_{n}\right) \bar{\varphi}\right|$, it follows that

$$
\left|w_{j-1} \wedge w_{j}\right| \leq\left|u_{n} \bar{\varphi} \wedge\left(u_{n} v_{n}\right) \bar{\varphi}\right| .
$$

Applying Lemma 6.1(i) to $w_{j-1} \pi$ and $a_{j} \varphi$, we get

$$
\begin{aligned}
\left|w_{j-1}\right| & \leq\left|w_{j-1} \wedge w_{j}\right|+K_{0} N_{0}+N_{0}\left|\overline{a_{j} \varphi}\right| \leq\left|w_{j-1} \wedge w_{j}\right|+X_{0} \\
& \leq\left|u_{n} \bar{\varphi} \wedge\left(u_{n} v_{n}\right) \bar{\varphi}\right|+X_{0}<\left|u_{n} \bar{\varphi}\right|-n+X_{0}=\left|u_{n} \bar{\varphi}\right|-m,
\end{aligned}
$$

and so $\left|u_{m}^{\prime} \bar{\varphi}\right|-\left|\left(u_{m}^{\prime} v_{m}^{\prime}\right) \bar{\varphi}\right|=\left|u_{n} \bar{\varphi}\right|-\left|w_{j-1}\right|>m$.

Suppose that $\left|w_{j-1}\right|-\left|u_{n} \bar{\varphi} \wedge w_{j-1}\right|>X_{0}$. Since we have seen above that $\left|w_{j-1}\right| \leq\left|w_{j-1} \wedge w_{j}\right|+X_{0}$, we get $\left|u_{n} \bar{\varphi} \wedge w_{j-1}\right|<\left|w_{j-1} \wedge w_{j}\right|$, in contradiction with $\left|w_{j-1} \wedge w_{j}\right| \leq\left|u_{n} \bar{\varphi} \wedge\left(u_{n} v_{n}\right) \bar{\varphi}\right|<\left|u_{n} \bar{\varphi} \wedge w_{j-1}\right|$. Thus

$$
\left|\left(u_{m}^{\prime} v_{m}^{\prime}\right) \bar{\varphi}\right|-\left|u_{m}^{\prime} \bar{\varphi} \wedge\left(u_{m}^{\prime} v_{m}^{\prime}\right) \bar{\varphi}\right|=\left|w_{j-1}\right|-\left|u_{n} \bar{\varphi} \wedge w_{j-1}\right| \leq X_{0},
$$

and so (12) holds.

We prove that

$$
\forall m \in \mathbb{N} \exists u_{m}^{\prime \prime} v_{m}^{\prime \prime} \in L:\left|u_{m}^{\prime \prime} \bar{\varphi}\right|>m \text { and }\left|\left(u_{m}^{\prime \prime} v_{m}^{\prime \prime}\right) \bar{\varphi}\right| \leq X_{0}+N_{0} D_{\varphi} .
$$


Indeed, let $m \in \mathbb{N}$. We have in $\Gamma_{A}(G)$ geodesics

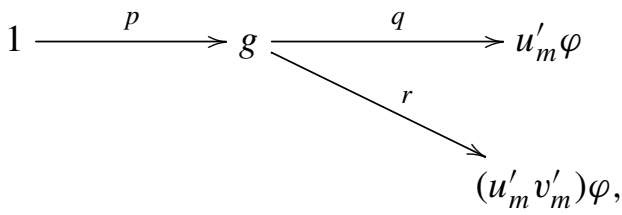

where $p q=u_{m}^{\prime} \bar{\varphi}, p r=\left(u_{m}^{\prime} v_{m}^{\prime}\right) \bar{\varphi}$, and $p=u_{m}^{\prime} \bar{\varphi} \wedge\left(u_{m}^{\prime} v_{m}^{\prime}\right) \bar{\varphi}$. Assume that $u_{m}^{\prime}=$ $a_{1} \cdots a_{k}\left(a_{i} \in A\right)$. Let

$I=\left\{i \in\{0, \ldots, k\}:\right.$ there exists a geodesic $\left(a_{1} \cdots a_{i}\right) \varphi \longrightarrow g \stackrel{q}{\longrightarrow} u_{m}^{\prime} \varphi$ in $\left.\Gamma_{A}(G)\right\}$.

Clearly, $0 \in I$. We claim that

$$
\left(i-1 \in I \text { and } d_{1}\left(\left(a_{1} \cdots a_{i-1}\right) \varphi, g\right)>N_{0} D_{\varphi}\right) \Rightarrow i \in I
$$

holds for $i=1, \ldots, k$. Indeed, assume $i-1 \in I$ and $\left(a_{1} \cdots a_{i-1}\right) \varphi \stackrel{y}{\longrightarrow} g \stackrel{q}{\longrightarrow} u_{m}^{\prime} \varphi$ is a geodesic with $y \in L$. Applying Lemma 5.1(ii) to the word $a_{i}^{-1} \bar{\varphi}$ and the geodesic $u=y q$, it follows that there exists some geodesic $\left(a_{1} \cdots a_{i}\right) \varphi \stackrel{z}{\longrightarrow} u_{m}^{\prime} \varphi$ such that $z$ and $u$ share a suffix of length $\geq|y q|-N_{0}\left|a_{i}^{-1} \bar{\varphi}\right| \geq|y q|-N_{0} D_{\varphi}>|q|$. Since $\Gamma_{A}(G)$ is deterministic, our geodesic $\left(a_{1} \cdots a_{i}\right) \varphi \stackrel{z}{\longrightarrow} u_{m}^{\prime} \varphi$ factors through $g$, and so (14) holds.

Since $k \notin I$ due to $|q|>0$, it follows from (14) that $d_{1}\left(\left(a_{1} \cdots a_{i}\right) \varphi, g\right) \leq N_{0} D_{\varphi}$ for some $i \in\{1, \ldots, k\}$. Let $j$ denote the smallest such $i$. We define $u_{m}^{\prime \prime}=a_{j+1} \cdots a_{k}$ and $v_{m}^{\prime \prime}=v_{m}^{\prime}$. Since $L$ is factorial and $u_{m}^{\prime} v_{m}^{\prime} \in L$, we also have $u_{m}^{\prime \prime} v_{m}^{\prime \prime} \in L$.

By minimality of $j$, we have $d_{1}\left(\left(a_{1} \cdots a_{i}\right) \varphi, g\right)>N_{0} D_{\varphi}$ for $i=0, \ldots, j-1$. By (14), we get $1, \ldots, j \in I$ and so there exists a geodesic $\left(a_{1} \cdots a_{j}\right) \varphi \longrightarrow g \stackrel{q}{\longrightarrow} u_{m}^{\prime} \varphi$ in $\Gamma_{A}(G)$. Hence

$$
\left|u_{m}^{\prime \prime} \bar{\varphi}\right|=d_{1}\left(1, u_{m}^{\prime \prime} \varphi\right)=d_{1}\left(\left(a_{1} \cdots a_{j}\right) \varphi, u_{m}^{\prime} \varphi\right) \geq|q| \geq\left|u_{m}^{\prime} \bar{\varphi}\right|-\left|\left(u_{m}^{\prime} v_{m}^{\prime}\right) \bar{\varphi}\right|>m .
$$

Finally,

$$
\begin{aligned}
\left|\left(u_{m}^{\prime \prime} v_{m}^{\prime \prime}\right) \bar{\varphi}\right| & =d_{1}\left(1,\left(u_{m}^{\prime \prime} v_{m}^{\prime \prime}\right) \varphi\right)=d_{1}\left(\left(a_{1} \cdots a_{j}\right) \varphi,\left(u_{m}^{\prime} v_{m}^{\prime}\right) \varphi\right) \\
& \leq d_{1}\left(\left(a_{1} \cdots a_{j}\right) \varphi, g\right)+d_{1}\left(g,\left(u_{m}^{\prime} v_{m}^{\prime}\right) \varphi\right) \leq N_{0} D_{\varphi}+|r| \\
& =N_{0} D_{\varphi}+\left|\left(u_{m}^{\prime} v_{m}^{\prime}\right) \bar{\varphi}\right|-\left|u_{m}^{\prime} \bar{\varphi} \wedge\left(u_{m}^{\prime} v_{m}^{\prime}\right) \bar{\varphi}\right| \leq N_{0} D_{\varphi}+X_{0}
\end{aligned}
$$

and so (13) holds.

Now, since $\left|\left(u_{m}^{\prime \prime} v_{m}^{\prime \prime}\right) \bar{\varphi}\right|$ is bounded, $u_{m}^{\prime \prime} v_{m}^{\prime \prime} \in L$, and $\operatorname{Ker} \varphi$ is finite, $\left|u_{m}^{\prime \prime} v_{m}^{\prime \prime}\right|$ must be bounded and so must be $\left|u_{m}^{\prime \prime}\right|$. This implies that $\left|u_{m}^{\prime \prime} \bar{\varphi}\right|$ must be bounded, contradicting $\left|u_{m}^{\prime \prime} \bar{\varphi}\right|>m$. Thus $\varphi$ satisfies the bounded cancellation property.

Proposition 7.2. The following conditions are equivalent for a nontrivial endomorphism $\varphi$ of $G$ : 
(i) $\varphi$ is uniformly continuous for $d_{2}$;

(ii) $\varphi$ is virtually injective.

Proof. (i) $\Rightarrow$ (ii). Suppose that $\operatorname{Ker} \varphi$ is infinite. In view of (10), it suffices to show that there exists some $\eta>0$ such that

$$
\forall \xi>0 \exists g, h \in G:(\rho(g, h)<\xi \text { and } \rho(g \varphi, h \varphi) \geq \eta) .
$$

By (10), we only need to show that there exists some $M \in \mathbb{N}$ such that

$$
\forall N \in \mathbb{N} \exists g, h \in G:((g \mid h)>N \text { and } g \varphi \neq h \varphi \text { and }((g \varphi) \mid(h \varphi)) \leq M) .
$$

Take $M=\left(g_{0} \varphi \mid 1\right)=0$ and fix $g_{0} \in G \backslash \operatorname{Ker} \varphi$. We prove the claim by showing that

$$
\forall N \in \mathbb{N} \exists h \in \operatorname{Ker} \varphi:\left(\left(h g_{0}\right) \mid h\right)>N .
$$

Let $N \in \mathbb{N}$. By Lemma 6.1(iii), we have $\left|\overline{h g_{0}} \wedge \bar{h}\right| \leq\left(\left(h g_{0}\right) \mid h\right)$ for every $h \in G$; hence we only need to find out $h \in \operatorname{Ker} \varphi$ satisfying $\left|\overline{h g_{0}} \wedge \bar{h}\right|>N$. By Lemma 6.1(i), we have $\left|\overline{h g_{0}} \wedge \bar{h}\right| \geq|\bar{h}|-K_{0} N_{0}-N_{0}\left|\overline{g_{0}}\right|$. Hence it suffices that $|\bar{h}|>N+K_{0} N_{0}+N_{0}\left|\overline{g_{0}}\right|$ for some $h \in \operatorname{Ker} \varphi$, and that is ensured by $\operatorname{Ker} \varphi$ being infinite. Thus (15) holds as required.

(ii) $\Rightarrow$ (i). Suppose that $\varphi$ is not uniformly continuous for $d_{2}$. In view of (10), there exists some $\eta>0$ such that

$$
\forall \xi>0 \exists g, h \in G:(\rho(g, h)<\xi \text { and } \rho(g \varphi, h \varphi) \geq \eta) .
$$

Hence, by (10), there exists some $M \in \mathbb{N}$ such that

$$
\forall N \in \mathbb{N} \exists g, h \in G:((g \mid h)>N \text { and } g \varphi \neq h \varphi \text { and }((g \varphi) \mid(h \varphi)) \leq M) .
$$

In view of Lemma 6.1(iii), we have that

$$
\forall n \in \mathbb{N} \exists u_{n}, v_{n} \in L:\left(\left|u_{n} \wedge v_{n}\right|>n \text { and } u_{n} \bar{\varphi} \neq v_{n} \bar{\varphi} \text { and }\left|u_{n} \bar{\varphi} \wedge v_{n} \bar{\varphi}\right| \leq M\right) .
$$

Let $w_{n}=u_{n} \wedge v_{n} \in L$. Then either $w_{n} \bar{\varphi} \neq u_{n} \bar{\varphi}$ or $w_{n} \bar{\varphi} \neq v_{n} \bar{\varphi}$. Without loss of generality, we may assume that $w_{n} \bar{\varphi} \neq u_{n} \bar{\varphi}$. Suppose that $\left|w_{n} \bar{\varphi}\right|>M+B_{\varphi}$. By definition of $B_{\varphi}$, we get $\left|w_{n} \bar{\varphi}\right|-\left|w_{n} \bar{\varphi} \wedge u_{n} \bar{\varphi}\right| \leq B_{\varphi}$, and so $\left|w_{n} \bar{\varphi} \wedge u_{n} \bar{\varphi}\right|>M$. Similarly, $\left|w_{n} \bar{\varphi} \wedge v_{n} \bar{\varphi}\right|>M$, and so $\left|u_{n} \bar{\varphi} \wedge v_{n} \bar{\varphi}\right|>M$, a contradiction. Therefore $\left|w_{n} \bar{\varphi}\right| \leq M+B_{\varphi}$ for every $n$. Since $\left|w_{n}\right|>n$ and $L$ is a cross-section for $\pi$, it follows that $\operatorname{Ker} \varphi$ is infinite.

Given a uniformly continuous endomorphism $\varphi$ of $\left(G, d_{2}\right), \bar{\varphi}: L \rightarrow L$ is uniformly continuous for $d_{3}$. Since $\hat{L}$ is the completion of $\left(L, d_{3}\right), \bar{\varphi}$ admits a unique continuous extension $\Phi: \hat{L} \rightarrow \hat{L}$. By continuity, we have

$$
\alpha \Phi=\left(\lim _{n \rightarrow+\infty} \alpha^{[n]}\right) \Phi=\lim _{n \rightarrow+\infty} \alpha^{[n]} \bar{\varphi} .
$$


Corollary 7.3. Let $\varphi$ be a uniformly continuous endomorphism of $G$ and $u \alpha \in \partial L$. Then $|u \bar{\varphi}|-|u \bar{\varphi} \wedge(u \alpha) \Phi| \leq B_{\varphi}$.

Proof. We have $(u \alpha) \Phi=\lim _{n \rightarrow+\infty}\left(u \alpha^{[n]}\right) \bar{\varphi}$ by (16). In view of Proposition 7.2, we have $\lim _{n \rightarrow+\infty}\left|\left(u \alpha^{[n]}\right) \bar{\varphi}\right|=+\infty$. Hence $|u \bar{\varphi} \wedge(u \alpha) \Phi|=\left|u \bar{\varphi} \wedge\left(u \alpha^{[m]}\right) \bar{\varphi}\right|$ for sufficiently large $m$. Since $u \alpha^{[m]} \in L$, the claim follows by definition of $B_{\varphi}$.

\section{Infinite fixed points}

Keeping all the notation and assumptions introduced in the preceding sections, we fix now a uniformly continuous endomorphism $\varphi$ of the finitely generated virtually free group $G$. We adapt notation introduced in [Ladra and Silva 2011] for free groups, and the proofs are adaptations of proofs in [Silva 2010].

Given $u \in L$, let $u \sigma=u \wedge u \bar{\varphi}$ and write

$$
u=(u \sigma)(u \tau), \quad u \bar{\varphi}=(u \sigma)(u \rho) .
$$

Also define

$$
u \sigma^{\prime}=\bigwedge\{(u v) \sigma: u v \in L\}
$$

and write $u \sigma=\left(u \sigma^{\prime}\right)\left(u \sigma^{\prime \prime}\right)$.

Lemma 8.1. Let $u v \in L$. Then

(i) $\left|u \sigma^{\prime \prime}\right| \leq B_{\varphi}$,

(ii) $|u \sigma|-|u \sigma \wedge(u v) \bar{\varphi}| \leq\left|u \sigma^{\prime \prime}\right|$,

(iii) $(u v) \bar{\varphi}=\left(u \sigma^{\prime}\right) \overline{\left(u \sigma^{\prime \prime}\right)(u \rho)(v \bar{\varphi})}$,

(iv) $(u v) \sigma^{\prime}=\left(u \sigma^{\prime}\right)\left(\bigwedge_{u v z \in L}\left(\overline{\left(u \sigma^{\prime \prime}\right)(u \rho)((v z) \bar{\varphi})} \wedge\left(u \sigma^{\prime \prime}\right)(u \tau) v z\right)\right)$.

$u v z \in L$

Proof. (i) We may assume that $|u \sigma|>B_{\varphi}$. Let $v$ denote the suffix of length $B_{\varphi}$ of $u \sigma$ and write $u \sigma=u^{\prime} v$. Suppose that $u w \in L$. It suffices to show that $u^{\prime}$ is a prefix of $(u w) \bar{\varphi}$, and this follows from

$$
\left|u^{\prime} v(u \rho)\right|-\left|u^{\prime} v(u \tau) \wedge(u w) \bar{\varphi}\right|=|u \bar{\varphi}|-|u \bar{\varphi} \wedge(u w) \bar{\varphi}| \leq B_{\varphi}
$$

and $|v|=B_{\varphi}$.

(ii) $u \sigma^{\prime}$ is a prefix of $u \sigma \wedge(u v) \bar{\varphi}$.

(iii) $u \sigma^{\prime}$ is a prefix of $(u v) \bar{\varphi}$ and both sides of the equality are equivalent in $G$.

(iv) $u \sigma^{\prime}$ is a prefix of $(u v) \sigma^{\prime}$ by (iii).

For every $u \in L$, we define

$$
u \xi=\left(u \sigma^{\prime \prime}, u \tau, u \rho, q_{0} u\right)
$$

Note that there exists precisely one path of the form $q_{0} \stackrel{u}{\longrightarrow} q_{0} u$ in $\mathscr{A}$. 
Lemma 8.2. Let $u, v \in L$ be such that $u \xi=v \xi$ and let $a \in \widetilde{A}, \alpha \in \widetilde{A}^{\infty}$. Then

(i) $u a \in L$ if and only if $v a \in L$;

(ii) if $u a \in L,(u a) \xi=(v a) \xi$;

(iii) $\overline{u v^{-1}} \in \operatorname{Fix} \bar{\varphi}$;

(iv) $u \alpha \in \hat{L}$ if and only if $v \alpha \in \hat{L}$;

(v) $u \alpha \in$ Fix $\Phi$ if and only if $v \alpha \in$ Fix $\Phi$;

(vi) if $\alpha \in \hat{L}, \alpha=\lim _{n \rightarrow+\infty} \overline{\alpha^{[n]} u}$.

Proof. (i) $u \xi=v \xi$ implies $q_{0} u=q_{0} v$.

(ii) Clearly, $q_{0} u=q_{0} v$ yields $q_{0} u a=q_{0} v a$. Considering $v=a$ in Lemma 8.1(iii), we may write $(u a) \sigma=\left(u \sigma^{\prime}\right) u^{\prime}$ and deduce that $u^{\prime},(u a) \tau$, and $(u a) \rho$ are all determined by $u \xi$. Hence $(u a) \tau=(v a) \tau,(u a) \rho=(v a) \rho$, and $u^{\prime}=v^{\prime}$.

Finally, since $q_{0} u=q_{0} v$, we have $u a z \in L$ if and only if $v a z \in L$. It follows from Lemma 8.1(iv) that there exists a word $x \in L$ which satisfies both $(u a) \sigma^{\prime}=$ $\left(u \sigma^{\prime}\right) x$ and $(v a) \sigma^{\prime}=\left(v \sigma^{\prime}\right) x$. Now $\left(u \sigma^{\prime}\right) u^{\prime}=(u a) \sigma=\left((u a) \sigma^{\prime}\right)\left((u a) \sigma^{\prime \prime}\right)=$ $\left(u \sigma^{\prime}\right) x\left((u a) \sigma^{\prime \prime}\right)$. Hence $u^{\prime}=x\left((u a) \sigma^{\prime \prime}\right)$. Similarly, $v^{\prime}=x\left((v a) \sigma^{\prime \prime}\right)$. Since $u^{\prime}=v^{\prime}$, we get $(u a) \sigma^{\prime \prime}=(v a) \sigma^{\prime \prime}$, and so $(u a) \xi=(v a) \xi$.

(iii)

$$
\begin{aligned}
\overline{\left(u v^{-1}\right) \varphi} & =\overline{(u \varphi)(v \varphi)^{-1}}=\overline{(u \sigma)(u \rho)(v \rho)^{-1}(v \sigma)^{-1}}=\overline{(u \sigma)(v \sigma)^{-1}} \\
& =\overline{(u \sigma)(u \tau)(v \tau)^{-1}(v \sigma)^{-1}}=\overline{u v^{-1}}
\end{aligned}
$$

(iv) We have $u \alpha \in \hat{L}$ if and only if $u \alpha^{[n]} \in L$ for every $n \in \mathbb{N}$. Now we use (i) and induction on $n$.

(v) We have $u \alpha=\left(u \sigma^{\prime}\right)\left(u \sigma^{\prime \prime}\right)(u \tau) \alpha$ and, in view of Corollary 7.3 and (16), also

$$
(u \alpha) \Phi=\left(u \sigma^{\prime}\right) \lim _{n \rightarrow+\infty} \overline{\left(u \sigma^{\prime \prime}\right)(u \rho)\left(\alpha^{[n]} \bar{\varphi}\right)} .
$$

Hence $u \alpha \in$ Fix $\Phi$ depends just on $u \xi$ and $\alpha$.

(vi) Let $m=K_{0} N_{0}+N_{0}|u|$. By Lemma 6.1(i), we have $\left|\alpha^{[n]} \wedge \overline{\alpha^{[n]} u}\right| \geq n-m$ for every $n$. Hence $\alpha=\lim _{n \rightarrow+\infty} \alpha^{[n-m]}=\lim _{n \rightarrow+\infty} \overline{\alpha^{[n]} u}$.

Given $X \subseteq A^{\infty}$, write

Pref $X=\left\{u \in A^{*}: u \alpha \in X\right.$ for some $\left.\alpha \in A^{\infty}\right\}$. 
Recall the finite trim deterministic $\widetilde{A}$-automaton $\mathscr{A}=\left(Q, q_{0}, Q, E\right)$ recognizing $L$. We build a (possibly infinite) $\widetilde{A}$-automaton $\mathscr{A}_{\varphi}^{\prime}=\left(Q^{\prime}, q_{0}^{\prime}, T^{\prime}, E^{\prime}\right)$ by taking

$$
\begin{aligned}
& Q^{\prime}=\{u \xi: u \in \operatorname{Pref} \text { Fix } \Phi\}, \\
& q_{0}^{\prime}=1 \xi \\
& T^{\prime}=\left\{u \xi \in Q^{\prime}: u \tau=u \rho=1\right\}, \\
& E^{\prime}=\left\{(u \xi, a, v \xi) \in Q^{\prime} \times \widetilde{A} \times Q^{\prime}: v=u a \in \operatorname{Pref} \text { Fix } \Phi\right\} .
\end{aligned}
$$

We note that $\mathscr{A}_{\varphi}^{\prime}$ is deterministic by Lemma 8.2(ii) and is also accessible: if $u \in \operatorname{Pref}$ Fix $\Phi$, there exists a path $q_{0}^{\prime} \stackrel{u}{\longrightarrow} u \xi$, and so every vertex can be reached from the initial vertex.

Let $S$ denote the set of all vertices $q \in Q^{\prime}$ such that there exist at least two edges in $\mathscr{R}_{\varphi}^{\prime}$ leaving $q$. Let $Q^{\prime \prime}$ denote the set of all vertices $q \in Q^{\prime}$ such that there exists some path $q \longrightarrow p \in S \cup T^{\prime}$. We define $\mathscr{A}_{\varphi}^{\prime \prime}=\left(Q^{\prime \prime}, q_{0}^{\prime \prime}, T^{\prime \prime}, E^{\prime \prime}\right)$ by taking $q_{0}^{\prime \prime}=q_{0}^{\prime}$, $T^{\prime \prime}=T^{\prime}$, and $E^{\prime \prime}=E^{\prime} \cap\left(Q^{\prime \prime} \times \widetilde{A} \times Q^{\prime \prime}\right)$.

Lemma 8.3. $S$ is finite.

Proof. In view of Lemma 8.1, the unique components of $u \xi$ that may assume infinitely many values are $u \tau$ and $u \rho$. Moreover, we claim that

$$
u \tau \neq 1 \Rightarrow|u \rho| \leq B_{\varphi}
$$

holds for every $u \in \operatorname{Pref}$ Fix $\Phi$. Indeed, suppose that $u \tau \neq 1$ and $|u \rho|>B_{\varphi}$. Write $\alpha=u \beta$ for some $\alpha \in$ Fix $\Phi$. In view of Corollary 7.3, $|u \rho|>B_{\varphi}$ yields $|(u \beta) \Phi \wedge u \bar{\varphi}|>|u \sigma|$ and now $u \tau \neq 1$ yields $((u \beta) \Phi \wedge u \beta)=(u \bar{\varphi} \wedge u)=u \sigma$. Since $\beta \neq 1$, this contradicts $\alpha \in$ Fix $\Phi$. Therefore (17) holds.

It is also easy to see that

$$
|u \rho|>B_{\varphi} \Rightarrow u \xi \notin S
$$

for every $u \in \operatorname{Pref}$ Fix $\Phi$. Indeed, if $|u \rho|>B_{\varphi}$ and $a$ is the first letter of $u \rho$, then, by definition of $B_{\varphi},(u \sigma) a$ is a prefix of $(u \alpha) \Phi$ whenever $u \alpha \in$ Fix $\Phi$. Therefore any edge leaving $u \xi$ in $\mathscr{A}_{\varphi}^{\prime}$ must have label $a$, and so (18) holds.

In view of Proposition 7.2, we can define

$$
W_{0}=\max \left\{|u|: u \in L,|u \bar{\varphi}| \leq 2\left(B_{\varphi}+D_{\varphi}-1\right)\right\} .
$$

Let $Z_{0}=B_{\varphi}+N_{0}\left(K_{0}+W_{0}\right) D_{\varphi}$. To complete the proof, it suffices to prove that

$$
|u \tau|>Z_{0} \Rightarrow u \xi \notin S
$$

for every $u \in \operatorname{Pref}$ Fix $\Phi$.

Suppose that $|u \tau|>Z_{0}$ and

$$
(u \xi, a,(u a) \xi),(u \xi, b,(u b) \xi) \in E^{\prime}
$$


for some $u \in \operatorname{Pref}$ Fix $\Phi$, where $a, b \in \widetilde{A}$ are distinct. We have $(u a) \xi=v \xi$ for some $v \in \operatorname{Pref}$ Fix $\Phi$. By Lemma 8.2(v), we get $u a \alpha \in$ Fix $\Phi$ for some $\alpha \in \hat{L}$. By (16), we get $u a \alpha=\lim _{n \rightarrow+\infty}\left(u a \alpha^{[n]}\right) \bar{\varphi}$, and so $\left|\left(u a \alpha^{[n]}\right) \bar{\varphi}\right| \geq|u|$ for sufficiently large $n$. Let

$$
p=\min \left\{n \in \mathbb{N}:\left|\left(u a \alpha^{[n]}\right) \bar{\varphi}\right| \geq|u|\right\} .
$$

Note that $p>0$ since $|u \tau|>Z_{0}$ and by (17). Since $\left|\left(u a \alpha^{[p-1]}\right) \bar{\varphi}\right|<|u|$ by the minimality of $p$, we get

$$
\left|\left(u a \alpha^{[p]}\right) \bar{\varphi}\right| \leq\left|\left(u a \alpha^{[p-1]}\right) \bar{\varphi}\right|+D_{\varphi}<|u|+D_{\varphi} .
$$

On the other hand,

$$
|u|-\left|\left(u a \alpha^{[p]}\right) \bar{\varphi} \wedge u\right| \leq B_{\varphi} .
$$

Otherwise, by definition of $B_{\varphi}, u a \alpha$ and $(u a \alpha) \Phi$ would differ at position

$$
\left|\left(u a \alpha^{[p]}\right) \bar{\varphi} \wedge u\right|+1 \text {. }
$$

Similarly, $u b \beta \in$ Fix $\Phi$ for some $\beta \in \hat{L}$. Defining

$$
q=\min \left\{n \in \mathbb{N}:\left|\left(u b \beta^{[n]}\right) \bar{\varphi}\right| \geq|u|\right\},
$$

we get

$$
\left|\left(u b \beta^{[q]}\right) \bar{\varphi}\right|<|u|+D_{\varphi}
$$

and

$$
|u|-\left|\left(u b \beta^{[q]}\right) \bar{\varphi} \wedge u\right| \leq B_{\varphi} .
$$

Write $u=u_{1} u_{2}$ with $\left|u_{2}\right|=B_{\varphi}$. Then by (20) and (21) we may write $\left(u a \alpha^{[p]}\right) \bar{\varphi}=u_{1} x$ for some $x$ such that $|x|<B_{\varphi}+D_{\varphi}$. Similarly, (22) and (23) yield $\left(u b \beta^{[q]}\right) \bar{\varphi}=u_{1} y$ for some $x$ such that $|x|<B_{\varphi}+D_{\varphi}$. Writing $w=\overline{\left(\beta^{[q]}\right)^{-1} b^{-1} a \alpha^{[p]}}$, it follows that $w \varphi=\left(y^{-1} x\right) \pi$, and so $|w \bar{\varphi}| \leq 2\left(B_{\varphi}+D_{\varphi}-1\right)$. Hence $|w| \leq W_{0}$. Applying Lemma 6.1(i) to $g=\left(u b \beta^{[q]}\right) \pi$ and $h=w \pi$, we get

$$
\left|u b \beta^{[q]}\right| \leq\left|u b \beta^{[q]} \wedge u a \alpha^{[p]}\right|+N_{0}\left(K_{0}+|w|\right) \leq|u|+N_{0}\left(K_{0}+W_{0}\right),
$$

and so $q<N_{0}\left(K_{0}+W_{0}\right)$. Hence, in view of (17), we get

$$
\begin{aligned}
|u \tau| & =|u|-|u \sigma| \leq\left|\left(u b \beta^{[q]}\right) \bar{\varphi}\right|-|u \sigma| \leq|u \bar{\varphi}|+\left|\left(b \beta^{[q]}\right) \bar{\varphi}\right|-|u \sigma| \\
& \leq|u \rho|+N_{0}\left(K_{0}+W_{0}\right) D_{\varphi} \leq B_{\varphi}+N_{0}\left(K_{0}+W_{0}\right) D_{\varphi},
\end{aligned}
$$

contradicting $|u \tau|>Z_{0}$. Thus (19) holds and the lemma is proved. 
We say that an infinite fixed point $\alpha \in$ Fix $\Phi \cap \partial L$ is singular if $\alpha$ belongs to the topological closure $(\operatorname{Fix} \varphi)^{c}$ of Fix $\varphi$. Otherwise, $\alpha$ is said to be regular. We denote by Sing $\Phi$ (respectively Reg $\Phi$ ) the set of all singular (respectively regular) infinite fixed points of $\Phi$.

Theorem 8.4. Let $\varphi$ be a uniformly continuous endomorphism of a finitely generated virtually free group $G$. Then

(i) the automaton $\mathscr{A}_{\varphi}^{\prime \prime}$ is finite;

(ii) $L\left(\mathscr{A}_{\varphi}^{\prime \prime}\right)=$ Fix $\bar{\varphi}$;

(iii) $L_{\omega}\left(\mathscr{A}_{\varphi}^{\prime \prime}\right)=\operatorname{Sing} \Phi$.

Proof. (i) The set $T^{\prime}$ is finite and $S$ is finite by Lemma 8.3. On the other hand, by definition of $S$, there are only finitely many paths in $\mathscr{A}_{\varphi}^{\prime}$ of the form $v_{j}: p^{\prime} \longrightarrow q^{\prime}$ with $p^{\prime}, q^{\prime} \in S \cup T^{\prime} \cup\left\{q_{0}^{\prime}\right\}$ and no intermediate vertex in $S \cup T^{\prime} \cup\left\{q_{0}^{\prime}\right\}$. Now recall that $\mathscr{A}_{\varphi}^{\prime}$ is accessible. Hence every path of the form $q \stackrel{u}{\longrightarrow} p \in S \cup T^{\prime}$ can be extended to some path $q_{0}^{\prime} \stackrel{v}{\longrightarrow} q \stackrel{u}{\longrightarrow} p \in S \cup T^{\prime}$ which is itself a concatenation of the finitely many paths $v_{j}$. Therefore $Q^{\prime \prime}$ is finite and so is $\mathscr{A}_{\varphi}^{\prime \prime}$.

(ii) Every $u \in L$ labels at most a unique path $q_{0}^{\prime}=1 \xi \stackrel{u}{\longrightarrow} u \xi$ out of the initial vertex in $\mathscr{A}_{\varphi}^{\prime}$. On the other hand, if $q_{0}^{\prime}=1 \xi \stackrel{u}{\longrightarrow} q^{\prime}$ is a path in $\mathscr{A}_{\varphi}^{\prime}$, the fourth component of $\xi$ yields a path $q_{0} \stackrel{u}{\longrightarrow} q$ in $\mathscr{A}$, and so $u \in L$. Hence

$$
L\left(\mathscr{A}_{\varphi}^{\prime}\right)=\left\{u \in L: u \xi \in T^{\prime}\right\}=\{u \in L: u \tau=u \rho=1\}=\operatorname{Fix} \bar{\varphi} .
$$

Since $L\left(\mathscr{A}_{\varphi}^{\prime \prime}\right)=L\left(\mathscr{A}_{\varphi}^{\prime}\right)$, (ii) holds.

(iii) Let $\alpha \in L_{\omega}\left(\mathscr{A}_{\varphi}^{\prime \prime}\right)$. Then there exists some $q^{\prime \prime} \in Q^{\prime \prime}$ and some infinite sequence $\left(i_{n}\right)_{n}$ such that $q_{0}^{\prime \prime} \stackrel{\alpha^{[i n]}}{\longrightarrow} q^{\prime \prime}$ is a path in $\mathscr{A}_{\varphi}^{\prime \prime}$ for every $n$. Write $u=\alpha^{\left[i_{1}\right]}$ and let $v_{n}=\overline{\alpha^{\left[i_{n}\right]} u^{-1}}$. By Lemma 8.2(iii), we have $v_{n} \in$ Fix $\bar{\varphi}$ for every $n$. It follows from Lemma 8.2(vi) that $\alpha=\lim _{n \rightarrow+\infty} v_{n}$. Thus $\alpha \in \operatorname{Sing} \Phi$.

Conversely, let $\alpha \in \operatorname{Sing} \Phi$. Then we may write $\alpha=\lim _{n \rightarrow+\infty} v_{n}$ for some sequence $\left(v_{n}\right)_{n}$ in Fix $\bar{\varphi}$. Let $k \in \mathbb{N}$. For large enough $n$, we have $\alpha^{[k]}=v_{n}^{[k]}$, and so there is some path

$$
q_{0}^{\prime \prime} \stackrel{\alpha^{[k]}}{\longrightarrow} q_{k}^{\prime \prime} \stackrel{w}{\longrightarrow} t_{k}^{\prime \prime} \in T^{\prime \prime},
$$

where $\alpha^{[k]} w=v_{n}$. Thus $\alpha \in L_{\omega}\left(\mathscr{A}_{\varphi}^{\prime \prime}\right)$ as required.

Recall now the continuous extensions $\hat{\tau}_{u}: \hat{L} \rightarrow \hat{L}$ of the uniformly continuous mappings $\tau_{u}: L \rightarrow L: v \mapsto \overline{u v}$ defined for each $u \in L$ (see Proposition 6.3). As remarked before, this is equivalent to saying that the left action

$$
L \times \partial L \rightarrow \partial L:(u, \alpha) \mapsto \overline{u \alpha}
$$

is continuous. Identifying $L$ with $G$ and $\partial L$ with $\partial G$, we have a continuous action (on the left) of $G$ on $\partial G$. Clearly, this action restricts to a left action of Fix $\varphi$ on 
Fix $\Phi \cap \partial G$ : if $g \in$ Fix $\varphi$ and $\alpha \in$ Fix $\Phi \cap \partial G$ with $\alpha=\lim _{n \rightarrow+\infty} g_{n}\left(g_{n} \in G\right)$,

$$
\begin{aligned}
(g \alpha) \Phi & =\left(g \lim _{n \rightarrow+\infty} g_{n}\right) \Phi=\left(\lim _{n \rightarrow+\infty} g g_{n}\right) \Phi=\lim _{n \rightarrow+\infty}\left(g g_{n}\right) \varphi \\
& =\lim _{n \rightarrow+\infty}(g \varphi)\left(g_{n} \varphi\right)=(g \varphi) \lim _{n \rightarrow+\infty} g_{n} \varphi=g\left(\lim _{n \rightarrow+\infty} g_{n}\right) \Phi \\
& =g(\alpha \Phi)=g \alpha .
\end{aligned}
$$

Moreover, the (Fix $\varphi$ )-orbits of Sing $\Phi$ and $\operatorname{Reg} \Phi$ are disjoint: if $\alpha \in \operatorname{Sing} \Phi$, we can write $\alpha=\lim _{n \rightarrow+\infty} g_{n}$ with the $g_{n} \in$ Fix $\varphi$ and get $g \alpha=\lim _{n \rightarrow+\infty} g g_{n}$ with $g g_{n} \in$ Fix $\varphi$ for every $n$; hence $\alpha \in \operatorname{Sing} \Phi \Rightarrow g \alpha \in \operatorname{Sing} \Phi$ and the action of $g^{-1}$ yields the converse implication.

We can now prove the main result of this section.

Theorem 8.5. Let $\varphi$ be a uniformly continuous endomorphism of a finitely generated virtually free group $G$. Then $\operatorname{Reg} \Phi$ has finitely many (Fix $\varphi$ )-orbits.

Proof. Let $P$ be the set of all infinite paths $s_{0}^{\prime} \stackrel{a_{1}}{\longrightarrow} s_{1}^{\prime} \stackrel{a_{2}}{\longrightarrow} \cdots$ in $\mathscr{A}_{\varphi}^{\prime}$ such that

$$
s_{0}^{\prime} \in S \cup\left\{q_{0}\right\}, \quad s_{n}^{\prime} \notin S \cup\left\{q_{0}\right\} \text { for every } n>0, \quad s_{n}^{\prime} \neq s_{m}^{\prime} \text { whenever } n \neq m .
$$

By Lemma 8.3, there are only finitely many choices for $s_{0}^{\prime}$. Since $A$ is finite and $\mathscr{A}_{\varphi}^{\prime}$ is deterministic, there are only finitely many choices for $s_{1}^{\prime}$, and from that vertex onwards, the path is uniquely determined due to $s_{n}^{\prime} \notin S(n \geq 1)$. Hence $P$ is finite, and we may assume that it consists of paths $p_{i}^{\prime} \stackrel{\alpha_{i}}{\longrightarrow} \cdots$ for $i=1, \ldots, m$. Fix a path $q_{0}^{\prime} \stackrel{u_{i}}{\longrightarrow} p_{i}^{\prime}$ for each $i$ and let $X=\left\{u_{1} \alpha_{1}, \ldots, u_{m} \alpha_{m}\right\} \subseteq \partial L$. We claim that $X \subseteq \operatorname{Reg} \Phi$.

Let $i \in\{1, \ldots, m\}$ and write $\beta=u_{i} \alpha_{i}$. To show that $\beta \in$ Fix $\Phi$, it suffices to show that $\lim _{n \rightarrow+\infty} \beta^{[n]} \bar{\varphi}=\beta$. Let $k \in \mathbb{N}$. We must show that there exists some $r \in \mathbb{N}$ such that

$$
n \geq r \Rightarrow\left|\beta^{[n]} \bar{\varphi} \wedge \beta\right|>k
$$

In view of Proposition 7.2, there exists some $r>k$ such that

$$
n \geq r \Rightarrow\left|\beta^{[n]} \bar{\varphi}\right|>k+B_{\varphi} .
$$

Suppose that $\left|\beta^{[n]} \bar{\varphi} \wedge \beta\right| \leq k$ for some $n \geq r$. Then $\left|\beta^{[n]} \sigma\right| \leq k$. Since $k<r \leq n$, it follows that $\beta^{[n]} \tau \neq 1$. On the other hand, since $\left|\beta^{[n]} \bar{\varphi}\right|>k+B_{\varphi}$, we get $\left|\beta^{[n]} \rho\right|>B_{\varphi}$. In view of (17), this contradicts $\beta^{[n]} \xi \in Q^{\prime}$. Therefore (24) holds for our choice of $r$ and so $X \subseteq$ Fix $\Phi$. Since the path

$$
q_{0}^{\prime} \stackrel{\beta}{\longrightarrow} \cdots
$$

can visit only finitely often a given vertex, $\beta \notin L_{\omega}\left(\mathscr{A}_{\varphi}^{\prime \prime}\right)$, and so $X \subseteq \operatorname{Reg} \Phi$ by Theorem 8.4(iii). 
By the previous comments on (Fix $\varphi$ )-orbits, the (Fix $\varphi$ )-orbits of the elements of $X$ must be contained in Reg $\Phi$. We complete the proof of the theorem by proving the opposite inclusion.

Let $\beta \in \operatorname{Reg} \Phi$. By Theorem 8.4(iii), we have $\beta \notin L_{\omega}\left(\mathscr{A}_{\varphi}^{\prime \prime}\right)$, and so there exists a factorization $\beta=u \alpha$ and a path

$$
q_{0}^{\prime} \stackrel{u}{\longrightarrow} p^{\prime} \stackrel{\alpha}{\longrightarrow} \cdots
$$

in $\mathscr{A}_{\varphi}^{\prime}$ such that $p^{\prime}$ signals the last occurrence of a vertex from $S \cup\left\{q_{0}^{\prime}\right\}$. We claim that no vertex is repeated after $p^{\prime}$. Otherwise, since no vertex of $S$ appears after $p^{\prime}$, we would get a factorization of $p^{\prime} \stackrel{\alpha}{\longrightarrow} \cdots$ as

$$
p^{\prime} \stackrel{v}{\longrightarrow} q^{\prime} \stackrel{w}{\longrightarrow} q^{\prime} \stackrel{w}{\longrightarrow} \cdots
$$

and by Lemma 8.2(iii) and (iv) we would get $\left(u v w^{n} v^{-1} u^{-1}\right) \pi \in$ Fix $\varphi$ and

$$
\beta=\lim _{n \rightarrow+\infty} \overline{u v w^{n} v^{-1} u^{-1}}
$$

contradicting $\beta \in \operatorname{Reg} \Phi$. Thus no vertex is repeated after $p^{\prime}$, and so we must have $p^{\prime}=p_{i}^{\prime}$ and $\alpha=\alpha_{i}$ for some $i \in\{1, \ldots, m\}$. It follows that $\beta=u \alpha_{i}$. By Lemma 8.2(iii), we get

$$
\overline{u u_{i}^{-1}} \in \operatorname{Fix} \bar{\varphi},
$$

and we are done.

Theorem 8.5 is somehow a version for infinite fixed points of Theorem 4.1, which we proved before for finite fixed points. Note however that Sing $\Phi$ does not in general have finitely many (Fix $\varphi$ )-orbits since Sing $\Phi$ may be uncountable (take for instance the identity automorphism on a free group of rank 2).

Since every finite set is closed in a metric space, we obtain the following corollary from Theorem 8.5.

Corollary 8.6. Let $\varphi$ be a uniformly continuous endomorphism of a finitely generated virtually free group $G$ with Fix $\varphi$ finite. Then Fix $\Phi$ is finite.

\section{Classification of the infinite fixed points}

We can now investigate the nature of the infinite fixed points of $\Phi$ when $\varphi$ is an automorphism. Since, by Proposition 7.2, both $\varphi$ and $\varphi^{-1}$ are then uniformly continuous, they extend to continuous mappings $\Phi$ and $\Psi$ which turn out to be mutually inverse in view of the uniqueness of continuous extensions to the completion. Therefore $\Phi$ is a bijection. We say that $\alpha \in \operatorname{Reg} \Phi$ is

- an attractor if $\exists \varepsilon>0 \forall \beta \in \hat{L}:\left(d_{3}(\alpha, \beta)<\varepsilon \Rightarrow \lim _{n \rightarrow+\infty} \beta \Phi^{n}=\alpha\right)$;

- a repeller if $\exists \varepsilon>0 \forall \beta \in \hat{L}:\left(d_{3}(\alpha, \beta)<\varepsilon \Rightarrow \lim _{n \rightarrow+\infty} \beta \Phi^{-n}=\alpha\right)$. 
The latter amounts to saying that $\alpha$ is an attractor for $\Phi^{-1}$. There exist other types, but they do not occur in our context as we shall see.

We say that an attractor $\alpha \in \operatorname{Reg} \Phi$ is exponentially stable if

$$
\exists \varepsilon, k, \ell>0 \forall \beta \in \hat{L} \forall n \in \mathbb{N}:\left(d_{3}(\alpha, \beta)<\varepsilon \Rightarrow d_{3}\left(\alpha, \beta \Phi^{n}\right) \leq k 2^{-\ell n} d_{3}(\alpha, \beta)\right) .
$$

This is equivalent to saying that

$$
\begin{aligned}
\exists M, N, \ell>0 \forall \beta \in \hat{L} \forall n & \in \mathbb{N}: \\
& \left(|\alpha \wedge \beta|>M \Rightarrow\left|\alpha \wedge \beta \Phi^{n}\right|+N>\ell n+|\alpha \wedge \beta|\right) .
\end{aligned}
$$

A repeller $\alpha \in \operatorname{Reg} \Phi$ is exponentially stable if it is an exponentially stable attractor for $\Phi^{-1}$.

Theorem 9.1. Let $\varphi$ be an automorphism of a finitely generated virtually free group $G$. Then $\operatorname{Reg} \Phi$ contains only exponentially stable attractors and exponentially stable repellers.

Proof. Let $\alpha \in \operatorname{Reg} \Phi$ and write $\alpha=a_{1} a_{2} \cdots$ with $a_{i} \in \tilde{A}$. Then there exists a path

$$
1 \xi \stackrel{a_{1}}{\longrightarrow} \alpha^{[1]} \xi \stackrel{a_{2}}{\longrightarrow} \alpha^{[2]} \xi \stackrel{a_{3}}{\longrightarrow} \cdots
$$

in $\mathscr{A}_{\varphi}^{\prime}$. Let $Y_{0}=B_{\varphi}\left(D_{\varphi^{-1}}+1\right)+B_{\varphi^{-1}}\left(D_{\varphi}+1\right)$ and let

$$
V=\left\{u \xi \in Q^{\prime}:|u \tau|>Y_{0} \text { or }|u \rho|>Y_{0}\right\} .
$$

It is easy to see that $Q^{\prime} \backslash V$ is finite. We saw in the proof of Theorem 8.5 that there are only finitely many repetitions of vertices in a path in $\mathscr{A}_{\varphi}^{\prime}$ labeled by a regular fixed point. Hence there exists some $n_{0} \in \mathbb{N}$ such that

$$
\alpha^{[n]} \xi \in V \text { for every } n \geq n_{0} .
$$

Now we consider two cases.

Case I: $\alpha^{\left[n_{0}\right]} \tau=1$. We claim that

$$
\alpha^{[n]} \tau=1 \text { for every } n \geq n_{0} .
$$

The case $n=n_{0}$ holds in Case I, so assume that $\alpha^{[n]} \tau=1$ for some $n \geq n_{0}$. Then $\alpha^{[n]} \xi \in V$, and so $\left|\alpha^{[n]} \rho\right|>Y_{0}>2 B_{\varphi}$. Since $\left|\alpha^{[n+1]} \bar{\varphi}\right| \geq\left|\alpha^{[n]} \bar{\varphi}\right|-B_{\varphi}$ by definition of $B_{\varphi}$,

$$
\begin{aligned}
\left|\alpha^{[n+1]} \rho\right| & \geq\left|\alpha^{[n+1]} \bar{\varphi}\right|-\left|\alpha^{[n+1]}\right| \geq\left|\alpha^{[n]} \bar{\varphi}\right|-B_{\varphi}-\left|\alpha^{[n]}\right|-1=\left|\alpha^{[n]} \rho\right|-B_{\varphi}-1 \\
& >Y_{0}-B_{\varphi}-1>B_{\varphi} .
\end{aligned}
$$

By (17), we get $\alpha^{[n+1]} \tau=1$, and so (27) holds.

Next we show that

$$
\left(\left(\alpha^{[n]} \gamma\right) \Phi\right)^{[n+1]}=\alpha^{[n+1]}
$$


if $n \geq n_{0}$ and $\alpha^{[n]} \gamma \in \hat{L}$. Indeed, by (27) we have $\alpha^{[n]} \bar{\varphi}=\alpha^{[n]}\left(\alpha^{[n]} \rho\right)$ and $\left|\alpha^{[n]} \rho\right|>$ $Y_{0}>B_{\varphi}$. By the definition of $B_{\varphi}$ and Corollary 7.3, we get $\left(\left(\alpha^{[n]} \gamma\right) \Phi\right)^{[n+1]}=$ $\alpha^{[n]}\left(\alpha^{[n]} \rho\right)^{[1]}$. Considering the particular case $\gamma=a_{n+1}$, we also get

$$
\left(\alpha^{[n+1]} \bar{\varphi}\right)^{[n+1]}=\alpha^{[n]}\left(\alpha^{[n]} \rho\right)^{[1]}=\left(\left(\alpha^{[n]} \gamma\right) \Phi\right)^{[n+1]} .
$$

Since $\alpha^{[n+1]} \tau=1$ by (27), we have $\left(\alpha^{[n+1]} \bar{\varphi}\right)^{[n+1]}=\alpha^{[n+1]}$, and so (28) holds.

Hence we may write $\left(\alpha^{[n]} \gamma\right) \Phi=\alpha^{[n+1]} \gamma^{\prime}$ whenever $\alpha^{[n]} \gamma \in \hat{L}$. Iterating, it follows that, for all $k \geq n_{0}$ and $n \in \mathbb{N}, \alpha^{[k]} \gamma \in \hat{L}$ implies $\left(\alpha^{[k]} \gamma\right) \Phi^{n}=\alpha^{[k+n]} \gamma^{\prime}$ for some $\gamma^{\prime}$. By considering $\beta=\alpha^{[k]} \gamma$ and $\alpha^{[k]}=\alpha \wedge \beta$, we deduce that

$$
|\alpha \wedge \beta| \geq n_{0} \Rightarrow\left|\alpha \wedge \beta \Phi^{n}\right| \geq n+|\alpha \wedge \beta|
$$

holds for all $\beta \in \hat{L}$ and $n \in \mathbb{N}$. Therefore (25) holds, and so $\alpha$ is an exponentially stable attractor in this case.

Now, if $\left|\alpha^{[t]} \tau\right|=1$ for some $t>n_{0}$, we can always replace $n_{0}$ by $t$ and deduce by Case I that $\alpha$ is an exponentially stable attractor. Thus we may assume the following.

Case II: $\alpha^{[n]} \tau \neq 1$ for every $n \geq n_{0}$. By replacing $n_{0}$ by a larger integer if necessary, we may assume that (26) is also satisfied when we consider the equivalents of $\xi$ and $V$ for $\varphi^{-1}$.

Since $\varphi$ is injective, there exists some $n_{1} \geq n_{0}$ such that

$$
\left|\alpha^{\left[n_{1}\right]} \bar{\varphi}\right| \geq n_{0}+B_{\varphi} .
$$

Since $\alpha^{\left[n_{1}\right]} \tau \neq 1$, it follows from (17) that $\left|\alpha^{\left[n_{1}\right]} \rho\right| \leq B_{\varphi}$; hence $\alpha^{\left[n_{1}\right]} \sigma=\alpha^{\left[n_{2}\right]}$ for some $n_{2} \geq n_{0}$. Write $x=\alpha^{\left[n_{1}\right]} \rho$. Then $\alpha^{\left[n_{1}\right]} \bar{\varphi}=\alpha^{\left[n_{2}\right]} x$ yields

$$
\alpha^{\left[n_{1}\right]}=\overline{\left(\alpha^{\left[n_{2}\right]} \overline{\varphi^{-1}}\right)\left(x \overline{\varphi^{-1}}\right)},
$$

and so

$$
n_{1}=\left|\alpha^{\left[n_{1}\right]}\right| \leq\left|\alpha^{\left[n_{2}\right]} \overline{\varphi^{-1}}\right|+\left|x \overline{\varphi^{-1}}\right| \leq\left|\alpha^{\left[n_{2}\right]} \overline{\varphi^{-1}}\right|+B_{\varphi} D_{\varphi^{-1}} .
$$

On the other hand, $\left|\alpha^{\left[n_{1}\right]} \rho\right| \leq B_{\varphi}<Y_{0}$ and $\alpha^{\left[n_{1}\right]} \in V$ together yield $Y_{0}<\left|\alpha^{\left[n_{1}\right]} \tau\right|=$ $n_{1}-n_{2}$, and so

$$
n_{2}+B_{\varphi^{-1}}<n_{1}-Y_{0}+B_{\varphi^{-1}}<n_{1}-B_{\varphi} D_{\varphi^{-1}} \leq\left|\alpha^{\left[n_{2}\right]} \overline{\varphi^{-1}}\right| .
$$

In view of (17), we can apply Case I to $\varphi^{-1}$. Hence $\alpha$ is an exponentially stable attractor for $\varphi^{-1}$ and, therefore, an exponentially stable repeller for $\varphi$.

\section{Example and open problems}

We include a simple example which illustrates some of the constructions introduced earlier. 
Example. Let $G=\mathbb{Z} \times \mathbb{Z}_{2}$ and let $A=\{a, b, c\}$. We note that this is not the canonical set of generators, which would not work. Then the matched homomorphism $\pi: \widetilde{A}^{*} \rightarrow G$ defined by

$$
a \pi=(1,0), \quad b \pi=(0,1), \quad c \pi=(1,1)
$$

yields

$$
\operatorname{Geo}_{A}(G)=(a \cup c)^{*} \cup\left(a^{-1} \cup c^{-1}\right)^{*} \cup\left\{b, b^{-1}\right\},
$$

and we can take

$$
\begin{array}{r}
\mathscr{R}=\left\{\left(x x^{-1}, 1\right): x \in \widetilde{A}\right\} \cup\left\{\left(a^{\varepsilon} b^{\delta}, c^{\varepsilon}\right),\left(b^{\delta} a^{\varepsilon}, c^{\varepsilon}\right),\left(c^{\varepsilon} b^{\delta}, a^{\varepsilon}\right),\left(b^{\delta} c^{\varepsilon}, a^{\varepsilon}\right): \delta, \varepsilon= \pm 1\right\} \\
\cup\left\{\left(a c^{-1}, b\right),\left(c^{-1} a, b\right),\left(a^{-1} c, b\right),\left(c a^{-1}, b\right),\left(b^{2}, 1\right),\left(b^{-2}, 1\right)\right\}
\end{array}
$$

to get $\operatorname{Geo}_{A}(G)=\operatorname{Irr} \mathscr{R}$. Ordering $\widetilde{A}$ by $a<c<a^{-1}<c^{-1}<b<b^{-1}$, we get

$$
L=a^{*}(1 \cup c) \cup\left(a^{-1}\right)^{*}\left(1 \cup c^{-1}\right) \cup b,
$$

recognized by the automaton $\mathscr{A}$ depicted by

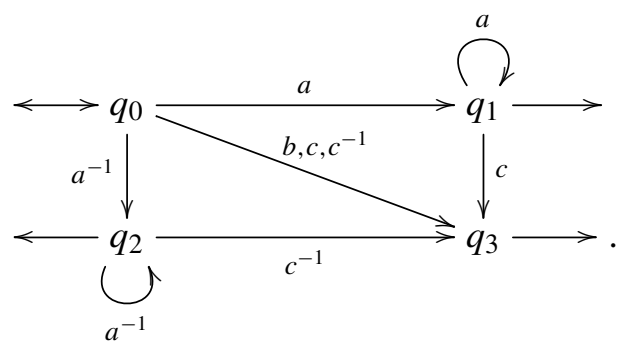

Hence $\partial L=L_{\omega}(\mathscr{A})=\left\{a^{\omega},\left(a^{-1}\right)^{\omega}\right\}$.

Let $\varphi$ be the endomorphism of $G$ defined by $(m, n) \varphi=(2 m, n)$. Then $\varphi$ is injective and therefore uniformly continuous, admitting a continuous extension $\Phi$ to $\hat{L}$. Since $B_{\varphi}=0$, it is easy to check that $\mathscr{A}_{\varphi}^{\prime}$ is the automaton

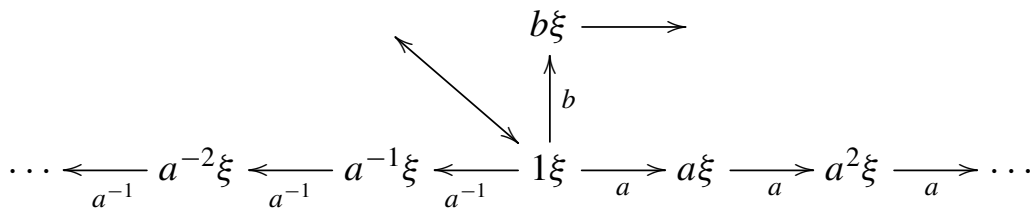

and

$1 \xi=\left(1,1,1, q_{0}\right), \quad b \xi=\left(1,1,1, q_{3}\right), a^{n} \xi=\left(1,1, a^{n}, q_{1}\right), \quad a^{-n} \xi=\left(1,1, a^{-n}, q_{2}\right)$

for $n \geq 1$. Note that, in general, we ignore how to compute $\mathscr{A}_{\varphi}^{\prime}$, our proofs being far from constructive!

It is immediate that Fix $\Phi=\left\{1, b, a^{\omega},\left(a^{-1}\right)^{\omega}\right\}$. Moreover, the regular infinite fixed points $a^{\omega}$ and $\left(a^{-1}\right)^{\omega}$ are both exponentially stable attractors. 
Finally, we end the paper with some easily predictable open problems.

Problem 10.1. Is it possible to generalize Theorems 4.1, 8.5, and 9.1 to arbitrary finitely generated hyperbolic groups?

Paulin proved [1989] that Theorem 4.1 holds for automorphisms of hyperbolic groups.

Problem 10.2. Is Fix $\varphi$ effectively computable when $\varphi$ is an endomorphism of a finitely generated virtually free group?

For the moment, only the case of free group automorphisms is known; see [Bogopolski and Maslakova 2012].

Another natural question to ask in this context is whether similar results hold for equalizers. Given homomorphisms $\varphi, \psi: G \rightarrow G^{\prime}$, let

$$
\operatorname{Eq}(\varphi, \psi)=\{x \in G: x \varphi=x \psi\}
$$

Problem 10.3. Given homomorphisms $\varphi, \psi: G \rightarrow G^{\prime}$ of finitely generated virtually free groups with $\varphi$ injective, is $\operatorname{Eq}(\varphi, \psi)$ finitely generated?

This question has been solved by Goldstein and Turner for free groups [1986]. The restriction to the case where at least one of the homomorphisms is injective is required even in the free group case (see [Gersten 1987] and [Ventura 2002, Section 3] for counterexamples).

\section{References}

[Berstel 1979] J. Berstel, Transductions and context-free languages, Leitfäden der Angewandten Mathematik und Mechanik 38, B. G. Teubner, Stuttgart, 1979. MR 80j:68056 Zbl 0424.68040

[Bestvina and Handel 1992] M. Bestvina and M. Handel, "Train tracks and automorphisms of free groups", Ann. of Math. (2) 135:1 (1992), 1-51. MR 92m:20017 Zbl 0757.57004

[Bogopolski and Maslakova 2012] O. Bogopolski and O. Maslakova, "A basis of the fixed point subgroup of an automorphism of a free group", preprint, 2012. arXiv 1204.6728

[Collins and Turner 1994] D. J. Collins and E. C. Turner, "Efficient representatives for automorphisms of free products", Michigan Math. J. 41:3 (1994), 443-464. MR 95k:20039 Zbl 0820.20035

[Cooper 1987] D. Cooper, "Automorphisms of free groups have finitely generated fixed point sets", $J$. Algebra 111:2 (1987), 453-456. MR 89a:20024 Zbl 0628.20029

[Dugundji 1966] J. Dugundji, Topology, Allyn and Bacon, Boston, MA, 1966. MR 33 \#1824 Zbl 0144.21501

[Epstein et al. 1992] D. B. A. Epstein, J. W. Cannon, D. F. Holt, S. V. F. Levy, M. S. Paterson, and W. P. Thurston, Word processing in groups, Jones and Bartlett, Boston, 1992. MR 93i:20036 Zbl 0764.20017

[Gaboriau et al. 1998] D. Gaboriau, A. Jaeger, G. Levitt, and M. Lustig, "An index for counting fixed points of automorphisms of free groups", Duke Math. J. 93:3 (1998), 425-452. MR 99f:20051 Zbl 0946.20010 
[Gersten 1987] S. M. Gersten, "Fixed points of automorphisms of free groups", Adv. in Math. 64:1 (1987), 51-85. MR 88f:20042 Zbl 0616.20014

[Ghys and de la Harpe 1990] E. Ghys and P. de la Harpe (editors), Sur les groupes hyperboliques d'après Mikhael Gromov, Progress in Mathematics 83, Birkhäuser, Boston, 1990. MR 92f:53050 Zbl 0731.20025

[Gilman et al. 2007] R. H. Gilman, S. Hermiller, D. F. Holt, and S. Rees, "A characterisation of virtually free groups", Arch. Math. (Basel) 89:4 (2007), 289-295. MR 2009a:20039 Zbl 1139.20029

[Goldstein and Turner 1985] R. Z. Goldstein and E. C. Turner, "Monomorphisms of finitely generated free groups have finitely generated equalizers", Invent. Math. 82:2 (1985), 283-289. MR 88b:57007 Zbl 0582.20023

[Goldstein and Turner 1986] R. Z. Goldstein and E. C. Turner, "Fixed subgroups of homomorphisms of free groups", Bull. London Math. Soc. 18:5 (1986), 468-470. MR 87m:20096 Zbl 0576.20016

[Ladra and Silva 2011] M. Ladra and P. V. Silva, "The generalized conjugacy problem for virtually free groups", Forum Math. 23:3 (2011), 447-482. MR 2012f:20101 Zbl 1230.20033

[Maslakova 2003] O. S. Maslakova, "The fixed point group of a free group automorphism", Algebra Logika 42:4 (2003), 422-472. In Russian; translated in Algebra and Logic 42 (2003), 237-265. MR 2004i:20044 Zbl 1031.20014

[Paulin 1989] F. Paulin, "Points fixes des automorphismes de groupe hyperbolique", Ann. Inst. Fourier (Grenoble) 39:3 (1989), 651-662. MR 91a:20030 Zbl 0674.20022

[Sakarovitch 2003] J. Sakarovitch, Eléments de Théorie des Automates, Vuibert, Paris, 2003. In French. Zbl 1178.68002

[Silva 2010] P. V. Silva, "Fixed points of endomorphisms over special confluent rewriting systems", Monatsh. Math. 161:4 (2010), 417-447. MR 2011k:20118 Zbl 1236.20063

[Silva 2012] P. V. Silva, "Fixed points of endomorphisms of certain free products", RAIRO Theor. Inform. Appl. 46:1 (2012), 165-179. MR 2904968 Zbl 06074750

[Sykiotis 2002] M. Sykiotis, "Fixed points of symmetric endomorphisms of groups", Internat. J. Algebra Comput. 12:5 (2002), 737-745. MR 2003k:20049 Zbl 1010.20016

[Sykiotis 2007] M. Sykiotis, "Fixed subgroups of endomorphisms of free products", J. Algebra 315:1 (2007), 274-278. MR 2008g:20052 Zbl 1130.20032

[Väisälä 2005] J. Väisälä, “Gromov hyperbolic spaces”, Expo. Math. 23:3 (2005), 187-231. MR 2006j:53055 Zbl 1087.53039

[Ventura 2002] E. Ventura, "Fixed subgroups in free groups: A survey", pp. 231-255 in Combinatorial and geometric group theory (New York, 2000/Hoboken, NJ, 2001), edited by S. Cleary et al., Contemp. Math. 296, Amer. Math. Soc., Providence, RI, 2002. MR 2003f:20037 Zbl 1025.20012

Received April 12, 2012. Revised November 7, 2012.

Pedro V. Silva

Centro de Matemática, Faculdade de Ciências

UNIVERSIDADE DO PORTO

R. CAMPo Alegre 687

4169-007 PORTO

PORTUGAL

pvsilva@fc.up.pt 


\title{
PACIFIC JOURNAL OF MATHEMATICS
}

\author{
msp.org/pjm
}

Founded in 1951 by E. F. Beckenbach (1906-1982) and F. Wolf (1904-1989)

\section{EDITORS}

V. S. Varadarajan (Managing Editor)

Department of Mathematics

University of California

Los Angeles, CA 90095-1555

pacific@math.ucla.edu

Paul Balmer

Department of Mathematics

University of California

Los Angeles, CA 90095-1555

balmer@math.ucla.edu

Daryl Cooper

Department of Mathematics

University of California

Santa Barbara, CA 93106-3080 cooper@math.ucsb.edu

Jiang-Hua $\mathrm{Lu}$

Department of Mathematics

The University of Hong Kong

Pokfulam Rd., Hong Kong jhlu@maths.hku.hk
Don Blasius

Department of Mathematics University of California

Los Angeles, CA 90095-1555

blasius@math.ucla.edu

Robert Finn

Department of Mathematics Stanford University

Stanford, CA 94305-2125

finn@math.stanford.edu

Sorin Popa

Department of Mathematics

University of California

Los Angeles, CA 90095-1555

popa@math.ucla.edu

Paul Yang

Department of Mathematics

Princeton University

Princeton NJ 08544-1000

yang@math.princeton.edu

\section{PRODUCTION}

Silvio Levy, Scientific Editor, production@msp.org

\section{SUPPORTING INSTITUTIONS}

ACADEMIA SINICA, TAIPEI

CALIFORNIA INST. OF TECHNOLOGY

INST. DE MATEMÁTICA PURA E APLICADA

KEIO UNIVERSITY

MATH. SCIENCES RESEARCH INSTITUTE

NEW MEXICO STATE UNIV.

OREGON STATE UNIV.

\author{
STANFORD UNIVERSITY \\ UNIV. OF BRITISH COLUMBIA \\ UNIV. OF CALIFORNIA, BERKELEY \\ UNIV. OF CALIFORNIA, DAVIS \\ UNIV. OF CALIFORNIA, LOS ANGELES \\ UNIV. OF CALIFORNIA, RIVERSIDE \\ UNIV. OF CALIFORNIA, SAN DIEGO \\ UNIV. OF CALIF., SANTA BARBARA
}

\author{
Vyjayanthi Chari \\ Department of Mathematics \\ University of California \\ Riverside, CA 92521-0135 \\ chari@math.ucr.edu \\ Kefeng Liu \\ Department of Mathematics \\ University of California \\ Los Angeles, CA 90095-1555 \\ liu@math.ucla.edu \\ Jie Qing \\ Department of Mathematics \\ University of California \\ Santa Cruz, CA 95064 \\ qing@cats.ucsc.edu
}

These supporting institutions contribute to the cost of publication of this Journal, but they are not owners or publishers and have no responsibility for its contents or policies.

See inside back cover or msp.org/pjm for submission instructions.

The subscription price for 2013 is US \$400/year for the electronic version, and \$485/year for print and electronic.

Subscriptions, requests for back issues and changes of subscribers address should be sent to Pacific Journal of Mathematics, P.O. Box 4163, Berkeley, CA 94704-0163, U.S.A. The Pacific Journal of Mathematics is indexed by Mathematical Reviews, Zentralblatt MATH, PASCAL CNRS Index, Referativnyi Zhurnal, Current Mathematical Publications and the Science Citation Index.

The Pacific Journal of Mathematics (ISSN 0030-8730) at the University of California, c/o Department of Mathematics, 798 Evans Hall \#3840, Berkeley, CA 94720-3840, is published monthly except July and August. Periodical rate postage paid at Berkeley, CA 94704, and additional mailing offices. POSTMASTER: send address changes to Pacific Journal of Mathematics, P.O. Box 4163, Berkeley, CA 94704-0163.

PJM peer review and production are managed by EditFLOW ${ }^{\circledR}$ from Mathematical Sciences Publishers.

PUBLISHED BY

mathematical sciences publishers

nonprofit scientific publishing

http://msp.org/

(C) 2013 Mathematical Sciences Publishers 


\section{PACIFIC JOURNAL OF MATHEMATICS}

Volume $263 \quad$ No. $1 \quad$ May 2013

Biharmonic hypersurfaces in complete Riemannian manifolds

Luis J. Alías, S. Carolina García-Martínez and Marco

RIGOLI

Half-commutative orthogonal Hopf algebras

JULIEN BiCHON and MiCHEL DUBOIS-ViOLETTE

Superdistributions, analytic and algebraic super Harish-Chandra pairs

Claudio CARMELI and RITA FIORESI

Orbifolds with signature $\left(0 ; k, k^{n-1}, k^{n}, k^{n}\right)$

Angel Carocca, Rubén A. Hidalgo and Rubí E.

RODRÍGUEZ

Explicit isogeny theorems for Drinfeld modules

IMIN CHEN and YOONJIN LEE

Topological pressures for $\epsilon$-stable and stable sets

XiAnfeng Ma and ERCAi Chen

Lipschitz and bilipschitz maps on Carnot groups

WILLIAM MEYERSON

Geometric inequalities in Carnot groups

FRANCESCOPAOLO MONTEFALCONE

Fixed points of endomorphisms of virtually free groups

PEDRO V. SILVA

The sharp lower bound for the first positive eigenvalue of the

Folland-Stein operator on a closed pseudohermitian $(2 n+1)$-manifold

CHIN-TUNG Wu

Remark on "Maximal functions on the unit $n$-sphere" by Peter M. Knopf 253 (1987)

HONG-QUAN LI 